\title{
CMC-SLICINGS OF KotTler-SchWARZSCHILD-DE SitTer Cosmologies
}

\author{
ROBERT BEIG* \\ Institute for Theoretical Physics, University of Vienna, \\ Boltzmanngasse 5, A-1090 Vienna, Austria \\ J. MARK HEINZLE ${ }^{\dagger}$ \\ Max-Planck-Institute for Gravitational Physics, \\ Am Mühlenberg 1, D-14476 Golm, Germany
}

\begin{abstract}
There is constructed, for each member of a one-parameter family of cosmological models, which is obtained from the Kottler-Schwarzschild-de Sitter spacetime by identification under discrete isometries, a slicing by spherically symmetric Cauchy hypersurfaces of constant mean curvature. These slicings are unique up to the action of the static Killing vector. Analytical and numerical results are found as to when different leaves of these slicings do not intersect, i.e. when the slicings form foliations.
\end{abstract}

Keywords: Constant mean curvature - CMC-slicing - CMC-foliation cosmological constant - Schwarzschild-de Sitter

\footnotetext{
*Electronic address: Robert.Beig@univie.ac.at
}

${ }^{\dagger}$ Electronic address: Mark.Heinzle@aei.mpg.de 


\section{Introduction}

Consider a globally hyperbolic spacetime which is spatially compact. One can ask the question whether there exists a slicing by Cauchy surfaces of constant mean curvature (in short: CMCslicing) and what are its properties. Since CMC-slicings have a wide variety of uses in general relativity, this question has received a lot of attention, we refer to 9] and [1] for recent overviews.

In the present paper we study this problem for a class of spatially compact spacetimes, which are quotient spaces of the Kottler-Schwarzschild-de Sitter family of spacetimes [7] under certain discrete subgroups of their isometry group. These spacetimes can be viewed as models of cosmological spacetimes containing a black hole. Studies of CMC-slicings which we are aware of treat cases where the spacetime has - in the future and/or past - either an all-encompassing crushing singularity or is geodesically complete, whereas in KottlerSchwarzschild-de Sitter both types of asymptotic behavior coexist. Furthermore, the KottlerSchwarzschild-de Sitter spacetimes, due to the presence of a positive cosmological constant, violate the so-called timelike convergence condition (strong energy condition) which most papers assume.

Before we state the main results of the present work, let us define and describe the family of Kottler-Schwarzschild-de Sitter cosmologies we consider:

The Kottler-Schwarzschild-de Sitter metric reads

$$
d s^{2}=-V d t^{2}+V^{-1} d r^{2}+r^{2} d \Omega^{2} \quad \text { where } \quad V=V(r)=1-\frac{2 M}{r}-\frac{\Lambda r^{2}}{3} .
$$

The cosmological constant $\Lambda>0$ and the constant $M>0$ are required to satisfy

$$
9 M^{2} \Lambda<1
$$

then the function $V(r)$ is positive in the interval $\left(r_{\mathrm{b}}, r_{\mathrm{c}}\right)$, and $V(r)=0$ at the "black hole horizon" $r=r_{\mathrm{b}}$ and at the "cosmological horizon" $r=r_{\mathrm{c}}$. The region $r_{\mathrm{b}}<r<r_{\mathrm{c}}$ is a static region of the spacetime with Killing vector $\xi=\partial_{t}$. (When $9 M^{2} \Lambda>1$, there are no horizons, the spacetime is not static but homogeneous.) It is straightforward to see that

$$
2 M<r_{\mathrm{b}}<3 M<\frac{1}{\sqrt{\Lambda}}<r_{\mathrm{c}}<\frac{3}{\sqrt{\Lambda}} .
$$

In the limit where $\Lambda$ goes to zero, the spacetime metric tends to Schwarzschild and $r_{\mathrm{b}}$ tends to $2 M$. In the limit where $M$ goes to zero, the metric becomes de Sitter and $r_{\mathrm{c}}$ tends to $3 / \sqrt{\Lambda}$.

The static region of the spacetime has an analytic extension reminiscent of the Kruskal extension of the Schwarzschild spacetime and the de Sitter spacetime. A common way of depicting the arising spacetime uses two charts which cover the regions $0<r<r_{\mathrm{c}}$ and $r_{\mathrm{b}}<r<\infty$. The conformal compactification of the region $0<r<r_{\mathrm{c}}$ is depicted in Fig. 1(a) it contains the curvature singularity $r=0$; the conformal compactification of $r_{\mathrm{b}}<r<\infty$ is shown in Fig. 1(b)] The overlap of the charts is $r_{\mathrm{b}}<r<r_{\mathrm{c}}$. As is wellknown, the constructed spacetime corresponding to the union of Figs. 1(a) and 1(b) can be 
smoothly (in fact analytically) extended in a "periodic-in-r"-fashion. Thereby one obtains an inextendible, globally hyperbolic spacetime of topology $\mathbb{R} \times \mathbb{R} \times S^{2}$ satisfying $G_{\mu \nu}+\Lambda g_{\mu \nu}=0$, see Fig. 2. We call this spacetime the Kottler-Schwarzschild-de Sitter spacetime KSSdS.

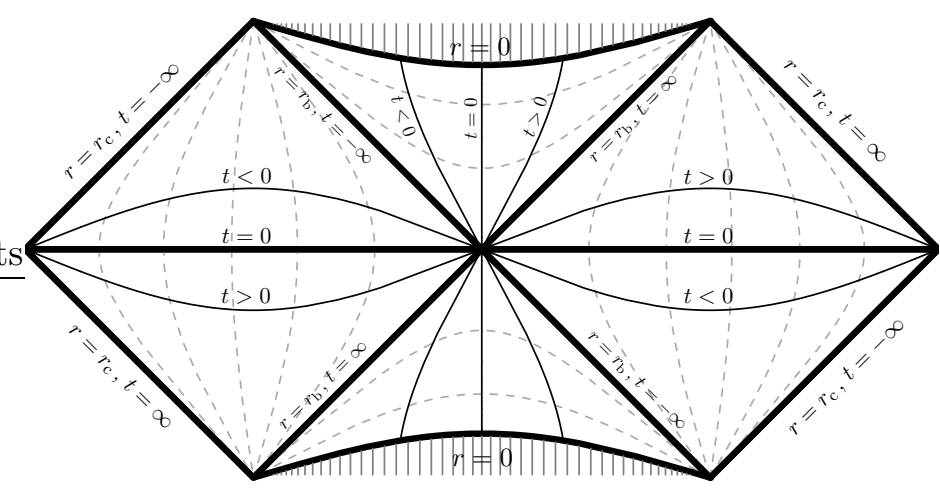

(a) $0<r<r_{\mathrm{c}}$

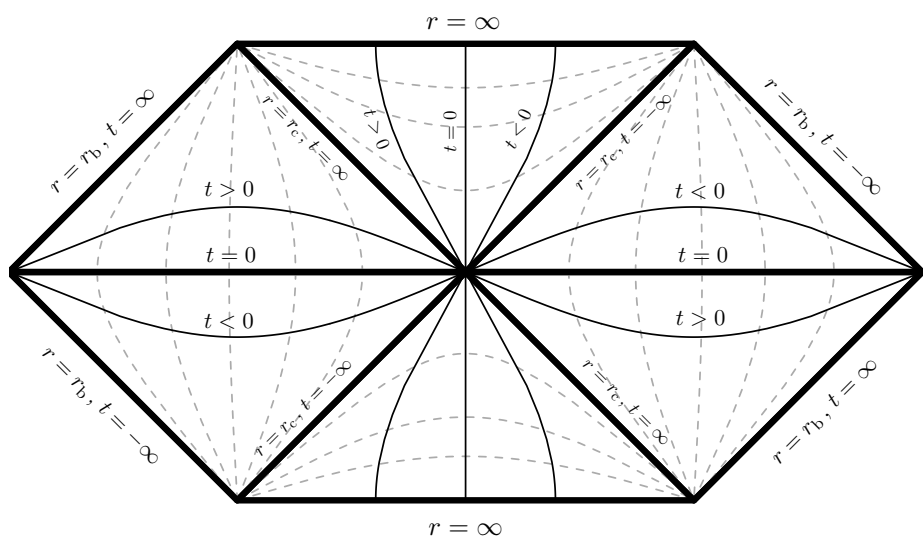

(b) $r_{\mathrm{b}}<r<\infty$

Figure 1: The figures show the compactified regions $0<r<r_{\mathrm{c}}$ and $r_{\mathrm{b}}<r<\infty$. Solid lines represent hypersurfaces $t=$ const, dashed lines are hypersurfaces $r=$ const.

On KSSdS there exists an isometric action of $\mathbb{R} \times \mathrm{SO}(3)$. The dashed lines in Figs. 1(a) and $1(\mathrm{~b})$ are orbits under the $\mathbb{R}$-factor in this action, i.e. under the static Killing vector $\xi=\partial_{t}$. Henceforth this action will be called "Killing flow" for brevity. Note that the Killing vector $\xi$ is globally defined; it is null on the Killing horizons $r=r_{\mathrm{b}}$ and $r=r_{\mathrm{c}}$ which emanate from the bifurcation 2 -spheres at which $\xi$ vanishes. Furthermore there exist discrete isometries ("reflections") leaving fixed the hypersurfaces $t=\mathrm{T}=$ const, which are given via $\mathrm{T}+t \mapsto \mathrm{T}-t$.

While $r$ is globally defined on KSSdS, $t$ blows up on the Killing horizons. In the static region or in the black hole, white hole, or cosmological regions, $(r, t)$ forms a coordinate system. The solid lines in Figs. 1(a) and 1(b) represent hypersurfaces $t=$ const, which are totally 
geodesic as fixed point sets of the discrete isometries. There exist two kinds: spacelike and timelike $t=$ const hypersurfaces; the latter we call $t=$ const cylinders.

By virtue of the periodicity in $r$, the $t=0$ cylinders in two adjoining copies of the region $0<r<r_{\mathrm{c}}$ can be identified, which results in a smooth spacetime of topology $\mathbb{R} \times S^{1} \times S^{2}$, which we call the cosmological Kottler-Schwarzschild-de Sitter spacetime KSSdS[0]. The spacetime KSSdS is the universal covering of $\operatorname{KSSdS}[0]$. More generally, let $\mathrm{T} \in \mathbb{R}$ and identify points of equal radius $r$ on a $t=0$ cylinder and a tilted $t=2 \mathrm{~T}$ cylinder in an adjacent copy of the region $0<r<r_{\mathrm{c}}$ on the r.h. side, see Fig. 2 Thereby we obtain a whole family of inextendible, globally hyperbolic, cosmological spacetimes, which we call KSSdS[T].

Note that KSSdS[T] is a smooth, in fact analytic, spacetime: consider a neighborhood of the $t=0$ cylinder in KSSdS. Via the Killing flow this neighborhood is isometric to a neighborhood of the $t=2 \mathrm{~T}$ cylinder, hence the hypersurfaces $t=0$ and $t=2 \mathrm{~T}$ agree not only in their induced first and second fundamental forms, but also in all higher derivatives of the fundamental forms. The identification of the two hypersurfaces thus results in a smooth manifold. Note that, while the spacetime KSSdS[0] is time-symmetric, KSSdS[T] with T $\neq 0$ is not; however, $\mathrm{KSSdS}[\mathrm{T}]$ and $\mathrm{KSSdS}[-\mathrm{T}]$ differ by time orientation only.

The spacetimes KSSdS[T] are not the only cosmological spacetimes which arise as quotient spaces from KSSdS. Firstly, we note that it is not necessary to identify $t=0$ with a $t=$ const cylinder in an adjoining copy of the region $0<r<r_{\mathrm{c}}$; indeed, an arbitrary number of intervening copies between the identified copies is possible. However, the arising spacetimes do not exhibit different structures as far as the properties of $\mathrm{CMC}$-slicings are concerned. Secondly, we may identify, in the black/white hole or in the future/past cosmological region, points mapped to each other by a discrete subgroup of the action under $\xi$. Thereby we obtain cosmological spacetimes that are completely different from the class KSSdS[T] considered here. These spacetimes will be treated in forthcoming work by one of us (J.M.H.) [5].

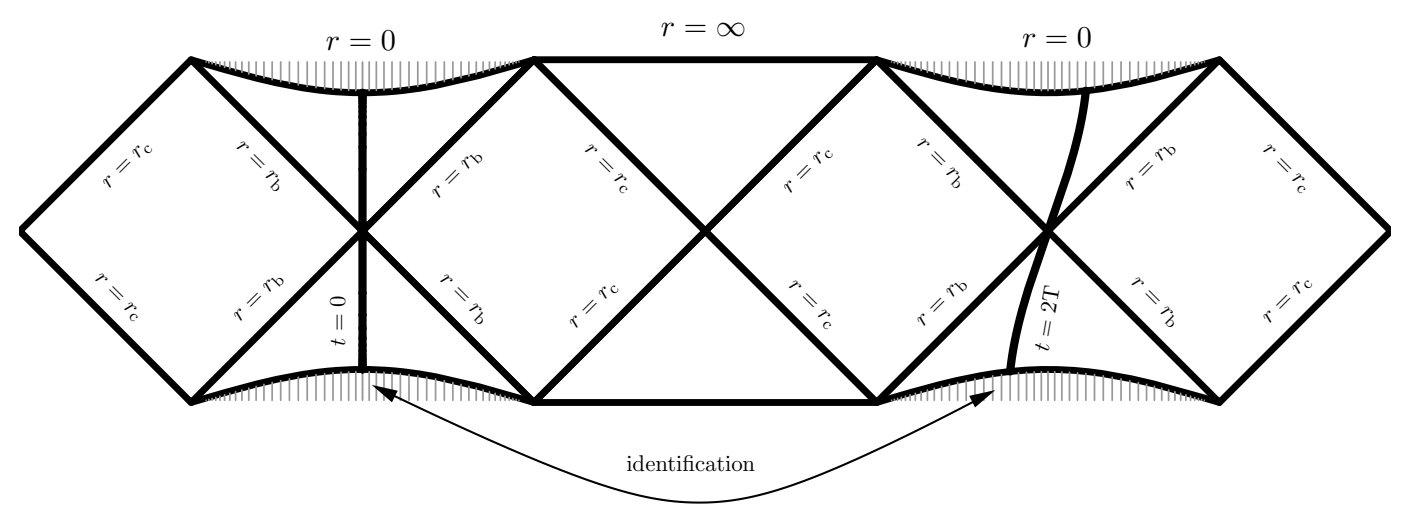

Figure 2: The compactified Kottler-Schwarzschild-de Sitter spacetime KSSdS[T].

In this paper, a slicing denotes a smooth family of smooth (spacelike) hypersurfaces. A parametrization of a slicing is a smooth map $\Psi: I \times \Sigma \rightarrow$ spacetime, where $\Sigma$ is a 3-manifold and $I \subseteq \mathbb{R}$, such that for all $\tau \in I, \Psi(\tau, \cdot)$ is an embedding. We require that $\Psi(\tau, \Sigma)$ is a hypersurface of the slicing for all $\tau \in I$, i.e. by the parametrization of the slicing the hypersurfaces are represented as level sets $\tau=$ const. Note that a slicing is a foliation iff the map $\Psi$ is a diffeomorphism onto its image. 
The content of the paper is as follows. In Sec. 2 we study spherically symmetric compact CMC-slices in terms of the associated initial data sets. We find that these compact CMCinitial data sets are parametrized by two constants $(K, C)$, which lie in a bounded connected open subset $\mathcal{K C}_{0}$ of $\mathbb{R}^{2}$. The constant $K$ is the mean curvature of the slice, i.e. the trace of the second fundamental form. The interpretation of the constant $C$ is less immediate (see the note following (6) $)$. Suffice it to say that initial data generated by $(K, C)$ is umbilical, i.e. the extrinsic curvature is proportional to the three-metric, iff $C=0$. The parameter space $\mathcal{K C}_{0}$ is investigated in some detail in Appendix $\mathrm{A}$. In Sec. 3 we discuss the embedding of the compact initial data sets into the cosmological spacetimes KSSdS[T]: we prove that each compact CMC-initial data set is embeddable as a Cauchy CMC-hypersurface into KSSdS[T] for a particular value of $\mathrm{T}$ and that the embedding is unique modulo the Killing flow. Secs. 4 and 5 are concerned with the formulation and the proof of the main results of the present paper.

One main theorem can be stated in an informal manner as follows: Each spacetime KSSdS[T] contains a unique non-trivial slicing of Cauchy CMC-hypersurfaces. (Here, a CMC-slicing is called trivial, if both $K$ and $C$ are constant along the slicing, and uniqueness is again understood modulo the Killing flow.) The first step in proving this theorem is undertaken in Sec. 4. where we show, using the implicit function theorem, that if the spacetime KSSdS[T] contains a compact CMC-hypersurface, then it evolves into a unique CMC-slicing. Crucial for this is the analysis of a (linear) ordinary differential equation, whose solution is interpreted as the lapse function of the slicing. Due to the violation of the timelike convergence condition, this analysis is quite involved and thus deferred to Appendix B. We find that the CMCslicing in KSSdS[T] can be represented by a curve in the parameter space $\mathcal{K C}_{0}$, which in turn is given as the $\mathrm{T}$-level set of a function $\mathcal{T}(K, C)$ on $\mathcal{K} \mathfrak{C}_{0}$. Thus there exists an interplay between a global but finite dimensional picture, where CMC-slicings are viewed in terms of their representation in $\mathcal{K C}_{0}$ on the one hand, and a local but essentially infinite dimensional view of slicings in spacetime on the other hand. Based on these ideas, in the second step in the proof of the theorem, in Sec. 5, we show that each spacetime KSSdS[T], T arbitrary, contains a compact $\mathrm{CMC}$-slicing and that this slicing is unique.

In another theorem in Sec. 5 we describe the asymptotic behavior of the CMC-slicings: Along each slicing $K$ tends to $\pm \sqrt{3 \Lambda}$ in the future resp. in the past, $C$ tends to explicitly known values that depend on $\Lambda$ and $M$. In spacetime the hypersurfaces of the slicing approach $r=(1 / \sqrt{\Lambda})[1-\sqrt{1-3 \sqrt{\Lambda} M}]$ in the black hole (resp. white hole), and $r=\infty$ in the future (resp. past) cosmological region (see Fig. 8). An essential ingredient in the proof of the theorem is an asymptotic analysis of the function $\mathcal{T}$.

Finally, in Sec. [5] and in Appendix [C] we discuss whether the compact CMC-slicings in the spacetimes KSSdS[T] are foliations. We prove that each slicing is a foliation at least during some time of its evolution. Moreover, if $|\mathrm{T}|$ is sufficiently large, then the slicing cannot be a foliation for all times. In addition, we provide solid numerical evidence that there are values for $(\Lambda, M)$ such that, if $|\mathrm{T}|$ is small, the CMC-slicing is a foliation everywhere.

In this work we can, and of course do, make use of the spherical symmetry of the spacetimes. The quantities we are seeking are all "essentially explicit" in this sense: they are either given by quadratures of algebraic functions, or solutions to algebraic equations, or level sets of functions which are in turn given by quadratures, and combinations of the above. Still only a 
small part of our task can be performed by a direct investigation of these expressions. Rather we require a somewhat delicate interplay between the analysis of the explicit quantities and the geometric analysis.

\section{CMC-data}

In this section we investigate spherically symmetric CMC-initial data sets. We find that there exists a two-parameter family of compact CMC-data; we analyze the parameter space, and we discuss the main geometric properties of the CMC-data sets in dependence on the parameters.

Consider a three-dimensional Riemannian manifold $\Sigma \cong J \times S^{2}$ endowed with a spherically symmetric 3 -metric

$$
{ }^{3} g_{i j} d x^{i} d x^{j}=d l^{2}+r^{2}(l)\left(d \vartheta^{2}+\sin ^{2} \vartheta d \varphi^{2}\right) ;
$$

the coordinates $\vartheta, \varphi$ are usual angular coordinates, $l$ is a "radial" coordinate which takes values in $J$, which is (an open interval of) $\mathbb{R}$, or $J \cong S^{1}$. Let there be given a symmetric tensor $K_{i j}$, the second fundamental form, such that the mean curvature

$$
K={ }^{3} g_{i j} K^{i j}=\text { const . }
$$

By (locally) solving, along the lines of [3], the vacuum constraints with positive cosmological constant $\Lambda$ we obtain that $\left(\Sigma,{ }^{3} g_{i j}, K_{i j}\right)$ is a spherically symmetric CMC-initial data set, iff there exists a constant $C$ such that

$$
K_{i j} d x^{i} d x^{j}=\left(\frac{K}{3}+\frac{2 C}{r(l)^{3}}\right) d l^{2}+\left(\frac{K}{3}-\frac{C}{r(l)^{3}}\right) r(l)^{2}\left(d \vartheta^{2}+\sin ^{2} \vartheta d \varphi^{2}\right) .
$$

Note that $K_{i j}$ is of the form $K_{i j}=(K / 3)^{3} g_{i j}+C L_{i j}^{\mathrm{TT}}$, i.e. it is the sum of a constant trace plus $C$ times a tensor that coincides with the unique spherically symmetric transverse traceless tensor w.r.t. ${ }^{3} g$. The initial data set is umbilical, i.e. $K_{i j} \sim g_{i j}$, iff $C=0$. The function $r(l)$ is required to satisfy

$$
r^{2}=1-\frac{2 M}{r}-\frac{\Lambda r^{2}}{3}+\left(\frac{K r}{3}-\frac{C}{r^{2}}\right)^{2}=: D(r),
$$

where the prime denotes the derivative w.r.t. $l$. The constant $M$ in (7) coincides with the mass $M$ appearing in (11), which is a consequence of the subsequent considerations.

An initial data set $\left(\Sigma,{ }^{3} g_{i j}, K_{i j}\right)$ is said to admit the Killing initial data (or KID) $\left(\alpha, X^{i}\right)$, if $\left(\alpha, X^{i}\right)$ lies in the kernel of the (overdetermined) operator given by the adjoint of the linearized constraint operator (see [4, also [2]). The KID-condition, in the presence of $\Lambda$, is equivalent to

$$
2 \alpha K_{i j}+2 D_{(i} X_{j)}=0
$$

together with

$$
D_{i} D_{j} \alpha+\mathcal{L}_{X} K_{i j}-\alpha\left({ }^{3} R_{i j}+K K_{i j}-2 K_{i}^{l} K_{j l}-\Lambda^{3} g_{i j}\right)=0,
$$


which, provided that $\alpha \neq 0$, is in turn equivalent to the statement that the "Killing development", i.e. the stationary metric

$$
g_{\mu \nu} d x^{\mu} d x^{\nu}=\left(-\alpha^{2}+X^{2}\right) d \tau^{2}+2 X_{i} d \tau d x^{i}+{ }^{3} g_{i j} d x^{i} d x^{j}
$$

satisfies $G_{\mu \nu}+\Lambda g_{\mu \nu}=0$. The present CMC-initial data set does in fact admit a KID, namely $\left(\alpha_{\xi}, X_{\xi}^{i}\right)$ given by

$$
\alpha_{\xi}=r^{\prime}(l) \quad, \quad X_{\xi}^{i} \partial_{i}=x_{\xi}(l) \frac{\partial}{\partial l}=-\left(\frac{K r(l)}{3}-\frac{C}{r(l)^{2}}\right) \frac{\partial}{\partial l} .
$$

The development of the initial data set thus results in a spacetime with Killing vector $\xi^{\mu}$,

$$
\xi^{\mu}=\alpha_{\xi} n^{\mu}+X_{\xi}^{\mu} \partial_{\mu} \quad, \quad \xi^{\mu} \xi_{\mu}=-\alpha_{\xi}^{2}+X_{\xi}^{2}=-\left(1-\frac{2 M}{r}-\frac{\Lambda r^{2}}{3}\right) .
$$

Here, $n^{\mu}$ denotes the unit normal of $\Sigma$ in the spacetime, $X_{\xi}^{2}=X_{\xi}^{i} X_{i}^{\xi}$. The KID is "static": from

$$
D_{[i}\left(\frac{X_{j]}^{\xi}}{-\alpha_{\xi}^{2}+X_{\xi}^{2}}\right)=0
$$

it follows that $\xi^{\mu}$ is hypersurface orthogonal. In the Killing development of the initial data, $\Sigma$ is given by $\tau=0$, and the metric reads

$$
g_{\mu \nu} d x^{\mu} d x^{\nu}=\left(-\alpha_{\xi}^{2}+X_{\xi}^{2}\right) d \tau^{2}+2 X_{i}^{\xi} d \tau d x^{i}+{ }^{3} g_{i j} d x^{i} d x^{j} .
$$

By using $r$ and introducing the coordinate $t$ through

$$
d \tau=d t-\frac{X_{i}^{\xi}}{-\alpha_{\xi}^{2}+X_{\xi}^{2}} d x^{i}=d t-V^{-1}\left(\frac{K r(l)}{3}-\frac{C}{r(l)^{2}}\right) d l
$$

we recover the original Schwarzschild-de Sitter metric (1),

$$
g_{\mu \nu} d x^{\mu} d x^{\nu}=-\left(1-\frac{2 M}{r}-\frac{\Lambda r^{2}}{3}\right) d t^{2}+\left(1-\frac{2 M}{r}-\frac{\Lambda r^{2}}{3}\right)^{-1} d r^{2}+r^{2} d \Omega^{2} .
$$

We record for later use the identity

$$
\nabla_{\mu} t=-V^{-1} \xi_{\mu}
$$

which follows from the previous discussion. As another particular consequence we conclude that the constant $M$ introduced in (17) is to be interpreted as the mass $M$ appearing in the Schwarzschild-de Sitter metric.

In the following we consider the cosmological constant $\Lambda$ and the mass $M$ as given constants. A CMC-initial data set $\left(\Sigma,{ }^{3} g_{i j}, K_{i j}\right)$ arises from the choice of a pair $(K, C)$, by which $D(r)$ and thus $r(l)$ is defined (modulo an irrelevant translational freedom in $l$ ), cf. (7)).

Definition. We define the parameter space of compact CMC-initial data by $\mathcal{K C}:=\left(\left\{(K, C) \mid(K, C) \text { generates compact CMC-data }\left(\Sigma,{ }^{3} g_{i j}, K_{i j}\right) \text { with } \Sigma \cong S^{1} \times S^{2}\right\}\right)^{\circ}$. 


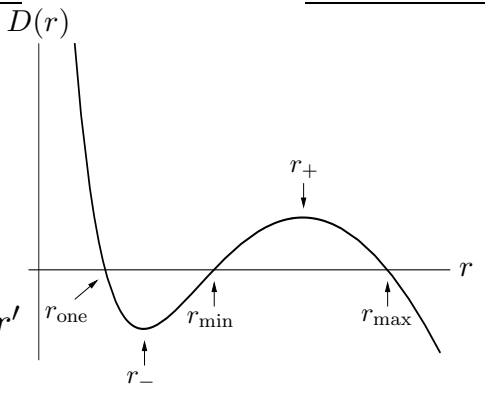

(a)

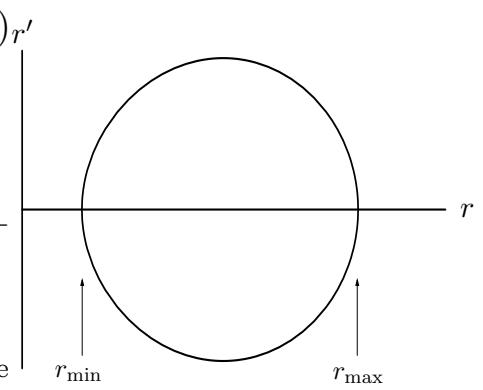

(b)

Figure 3: For $(K, C) \in \mathcal{K} \mathcal{C}, D(r)$ is positive between two positive zeros, $r_{\min } / r_{\max }$, and $d D / d r$ does not vanish at $r_{\min } / r_{\max }$. Then $D(r)$ gives rise to a (smooth) closed curve in the $\left(r, r^{\prime}\right)$-plane, and $r(l)$ becomes a periodic function.

Proposition 2.1. The parameter space $\mathcal{K} \mathcal{C}$ is the disjoint union of three open connected domains,

$$
\mathcal{K C}=\mathcal{K C}_{0} \cup \mathcal{K C}_{1} \cup \mathcal{K} \mathcal{C}_{-1}
$$

$\mathcal{K C}_{0}$ is the connection component of $(K, C)=(0,0)$, it is invariant under the inversion.

For proofs we refer to App. A. In this paper, we focus on compact CMC-initial data generated by $(K, C) \in \mathcal{K C}_{0}$. The space $\mathcal{K C}_{0}$ is depicted in Fig. [4 it is enclosed by the curves $C_{t}$ and $C_{b}$, and the vertical straight lines $K= \pm \sqrt{3 \Lambda}$. (In the following we refrain from making a distinction between the curves $C_{b, t}$ and the functions $C_{t}(K), C_{b}(K)=-C_{t}(-K)$, that parametrize the curves.) Some properties of the functions $C_{b, t}(K)$ are discussed in App. $\mathrm{A}$.

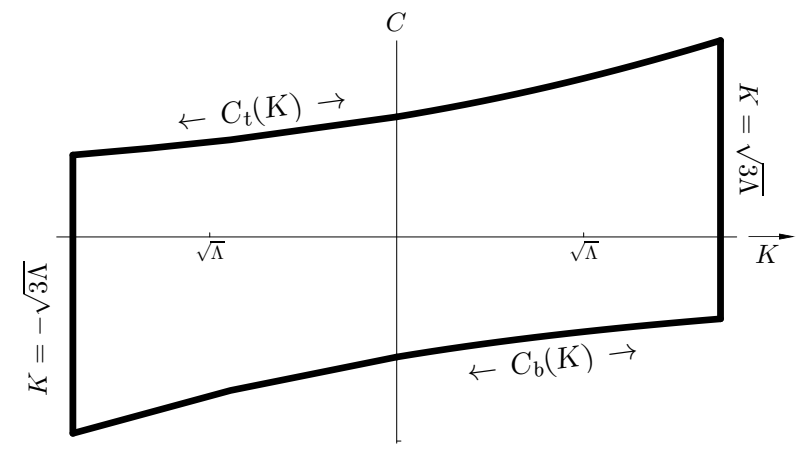

Figure 4: $\mathcal{K C}_{0}$ for $\Lambda=1, M=1 / 4$; it is enclosed by $C_{b, t}(K)$ and $K= \pm \sqrt{3 \Lambda}$.

If and only if $(K, C) \in \mathcal{K C}$, the function $D(r)$ exhibits the form depicted in Fig. 3(a) in particular, $D(r)$ possesses two positive (simple) zeros, $r_{\min }$ and $r_{\max }$, such that $D(r)>0$ in the interval $\left(r_{\min }, r_{\max }\right)$. Accordingly, when viewed over $r \in\left[r_{\min }, r_{\max }\right], r^{\prime}= \pm \sqrt{D(r)}$ describes a closed curve, cf. Fig. 3(b)] so that $r(l)$ becomes a periodic function that oscillates 
between $r_{\min }$ and $r_{\max }$; we denote the period by $2 L$,

$$
L=\int_{r_{\min }}^{r_{\max }} D^{-1 / 2}(r) d r .
$$

Without loss of generality we assume that

$$
r(0)=r_{\min } \quad \text { so that } r( \pm L)=r_{\max } ;
$$

it follows that $r(l)$ is an even periodic function, which is implicitly given through

$$
l(r)= \pm \int_{r_{\min }}^{r} D^{-1 / 2}(\hat{r}) d \hat{r} .
$$

By the natural identification of $l=-L$ and $l=L$, the domain of the function $r(l)$ becomes $S^{1}$, so that the CMC-initial data $\left(\Sigma,{ }^{3} g_{i j}, K_{i j}\right)$ is compact with $\Sigma \cong S^{1} \times S^{2}$.

Remark. From Fig. 3(a) we see that a pair $(K, C)$ that generates compact CMC-data, in general also gives rise to non-compact CMC-data, where $r(l)$ ranges in $\left(0, r_{\text {one }}\right]$. A CMCdata set of this type is embeddable in KSSdS as a hypersurface that runs into the singularity. A detailed classification of all possible types of CMC-data sets and their embeddings in $\mathrm{KSSdS}[\mathrm{T}]$ and other cosmological KSSdS-spacetimes will be presented in [5]

When $(K, C) \in \partial(\mathcal{K C})$, the profile of the function $D(r)$ is a borderline case of the profile 3(a) On the boundaries $C_{b, t}$, the function has the form 5(a) i.e. the zero $r_{\min }$ is a double zero. The solution of $r^{\prime}= \pm \sqrt{D(r)}$ that is relevant for our purposes is $r(l) \equiv$ const $=r_{\min }$ : by identifying $l=l_{0}$ with $l=l_{1}$ for any $l_{0}, l_{1}$, we obtain a compact CMC-initial data set $\left(\Sigma,{ }^{3} g_{i j}, K_{i j}\right), \Sigma \cong S^{1} \times S^{2}$. On the boundaries $K= \pm \sqrt{3 \Lambda}, D(r)$ possesses a simple zero $r_{\text {min }}$, but " $r_{\max }=\infty$ ", i.e. $D \rightarrow 1(r \rightarrow \infty)$, see Fig. 5(b). In this case, no compact CMC-data is generated. The features of $D(r)$ described above occur simultaneously on the intersections of the boundaries.

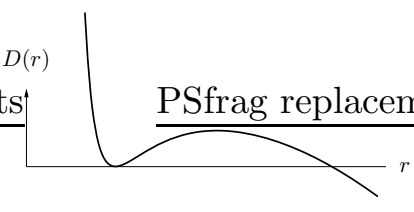

(a) $(K, C) \in C_{b, t}$

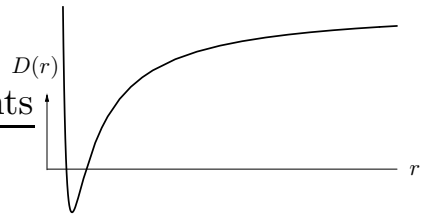

(b) $K= \pm \sqrt{3 \Lambda}$

Figure 5: The profiles of $D(r)$ on $\partial\left(\mathcal{K} \mathcal{C}_{0}\right)$.

For every compact CMC-initial data set $\left(S^{1} \times S^{2},{ }^{3} g_{i j}, K_{i j}\right)$ associated with $(K, C) \in \mathcal{K C}_{0}$, the radius $r$ of the spheres of symmetry varies between values $r_{\min }$ and $r_{\max }$. We conclude the section by discussing $r_{\min }(K, C)$ and $r_{\max }(K, C)$ in dependence on $(K, C) \in \overline{\mathcal{K C}_{0}}$.

Most importantly, we note that $r_{\min } \leq r_{\mathrm{b}}$ and that $r_{\min }=r_{\mathrm{b}}$ iff $(K, C)$ is such that $C=\left(r_{\mathrm{b}}^{3} / 3\right) K$. This "line of maximal $r_{\text {min }}$ " divides $\overline{\mathcal{K} \mathcal{C}_{0}}$ in two regions, an upper (left) half, 
characterized by $\left[K r_{\min } / 3-C / r_{\min }^{2}\right]<0$, and a lower (right) half with $\left[K r_{\min } / 3-C / r_{\min }^{2}\right]>0$, see Fig. $10(\mathrm{a})$ The function $r_{\text {min }}$ assumes its global minimum on $\overline{\mathcal{K C}_{0}}$ at the point $(K, C)=$ $\left(\sqrt{3 \Lambda}, C_{b}(\sqrt{3 \Lambda})\right)$ and at the reflected point; the minimal value is given by (23). $r_{\text {min }}$ decreases/increases along the boundaries of $\mathcal{K C}_{0}$ as depicted in Fig. 10(a). $r_{\max } \geq r_{\mathrm{c}}$ and $r_{\max }=r_{\mathrm{c}}$ iff $(K, C)$ is such that $C=\left(r_{\mathrm{c}}^{3} / 3\right) K$. This "line of minimal $r_{\max }$ " divides $\overline{\mathcal{K} \mathcal{C}_{0}}$ in two regions, an (upper) left half, where $\left[K r_{\max } / 3-C / r_{\max }^{2}\right]<0$ and a (lower) right half, where $\left[K r_{\max } / 3-C / r_{\max }^{2}\right]>0$, see Fig. [10(b) $r_{\max }$ is unbounded on $\mathcal{K} \mathcal{C}_{0}$, it diverges like $\sqrt{3} / \sqrt{\bar{\Lambda}}$ as $K \rightarrow \pm \sqrt{3 \Lambda} ; \bar{\Lambda}=\Lambda-K^{2} / 3$. Both $r_{\min }$ and $r_{\max }$ are constant along straight lines in $\mathcal{K C}_{0}$, see Fig. 11 .

$r_{\min }$ can be given explicitly,

$$
r_{\min }=\frac{1}{\sqrt{\Lambda}}( \pm 1 \mp \sqrt{1 \mp 3 \sqrt{\Lambda} M}), \quad \text { for } K=\sqrt{3 \Lambda}, C=C_{b, t}(\sqrt{3 \Lambda}) .
$$

Here, the upper sign applies to $C_{t}$, the lower to $C_{b}$.

\section{Embeddings}

In this section we investigate embeddings of compact CMC-initial data $\left(\Sigma,{ }^{3} g_{i j}, K_{i j}\right)$ as CMChypersurfaces in Schwarzschild-de Sitter spacetime.

Let $(K, C) \in \mathcal{K C}_{0}$; the pair generates compact CMC-data $\left(\Sigma \cong S^{1} \times S^{2},{ }^{3} g_{i j}, K_{i j}\right)$. We consider the universal covering $\left(\tilde{\Sigma} \cong \mathbb{R} \times S^{2},{ }^{3} g_{i j}, K_{i j}\right)$ of the data by regarding $r(l)$ as a periodic even function on $\mathbb{R}$. The initial data set $\left(\tilde{\Sigma},{ }^{3} g_{i j}, K_{i j}\right)$ is embedded as a CMC-hypersurface $\tilde{\mathcal{S}}$ in KSSdS via

$$
r=r(l) \quad t=t(l):=\int_{0}^{l} V^{-1}(r(\hat{l}))\left(\frac{K r(\hat{l})}{3}-\frac{C}{r(\hat{l})^{2}}\right) d \hat{l},
$$

which follows from (15). The integral is understood in the principal value sense, so that $t(l)$ is well-defined for all $l$ with $V(r(l)) \neq 0$; this suffices to uniquely define the embedded hypersurface in KSSdS. (Alternatively, the embedding can be given in Kruskal type coordinates.) If $(K, C)$ is such that $r_{\min }=r_{\mathrm{b}}$, then $V^{-1}$ is singular at $l=0$, but also $\left(K r / 3-C / r^{2}\right)=0$ when $l \rightarrow 0$, cf. Fig. 10(a). Using de l'Hospital's rule we see $\lim _{l \rightarrow 0} V^{-1}(r(l))\left[K r(l) / 3-C / r^{2}(l)\right]=$ $-V^{\prime-1}\left(r_{\mathrm{b}}\right)(K / 3)$, i.e. the integrand is bounded as $l \rightarrow 0$. Similarly, the integrand is always bounded as $l \rightarrow L$.

The CMC-hypersurface $\tilde{\mathcal{S}}$ defined by (24) is not the unique embedding of $\tilde{\Sigma}$ : integrating (15) leaves the freedom of choosing an integration constant so that $t$ is replaced by $t-$ const in (24). The one-parameter freedom is associated with the Killing flow, hence, modulo the Killing isometries the embedding is indeed unique.

Let $(K, C) \in \mathcal{K C}_{0}$. Then $r(l)$ oscillates between $r_{\min } \leq r_{\mathrm{b}}$ and $r_{\max } \geq r_{\mathrm{c}}$, therefore $\tilde{\mathcal{S}}$ is a Cauchy hypersurface in KSSdS, see Fig. [6]

The (future pointing) unit normal of $\tilde{\mathcal{S}}$ is given by

$$
n^{\mu} \partial_{\mu}=r^{\prime} V^{-1} \frac{\partial}{\partial t}+\left(\frac{K r}{3}-\frac{C}{r^{2}}\right) \frac{\partial}{\partial r}
$$




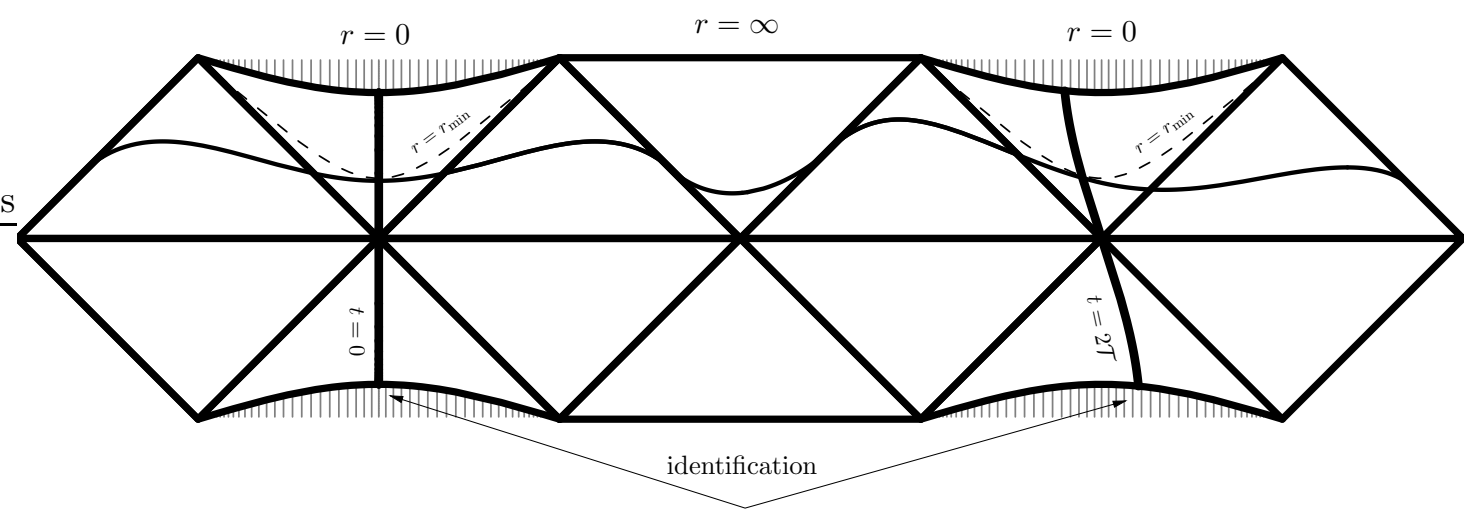

Figure 6: The embedding of the compact CMC-initial data with $K=1$ and $C=0.1$ in KSSdS[T] (with $\Lambda=1, M=1 / 4$ ). The hypersurface is not null at the horizon $r_{\mathrm{c}}$ : for the figure we have used the coordinate system of the region $0<r<r_{\mathrm{c}}$ and extended it to $0<r<\infty$ to obtain global coordinates; however, these coordinates break down at $r=r_{\mathrm{c}}$.

it is straightforward to check $\nabla_{\mu} n^{\mu}=K$. The CMC-hypersurface $\tilde{\mathcal{S}}$ passes through the white hole region [black hole region] if $\left(K r_{\min } / 3-C / r_{\min }^{2}\right)>0\left[\left(K r_{\min } / 3-C / r_{\min }^{2}\right)<0\right]$; when $\left(K r_{\min } / 3-C / r_{\min }^{2}\right)=0, r_{\min }=r_{\mathrm{b}}$ and the hypersurface passes through the bifurcation sphere; compare with Fig. 10(a). Similarly, the hypersurface passes through the future [past] cosmological region if $\left(K r_{\max } / 3-C / r_{\max }^{2}\right)>0\left[K r_{\max } / 3-C / r_{\max }^{2}<0\right]$, cf. Fig. 10(b)] These relations guarantee that $\tilde{\mathcal{S}}$ cannot oscillate between the black hole and the white hole (or the future and the past cosmological regions): either $\tilde{\mathcal{S}}$ passes through the black hole regions or through the white hole regions for all $l=2 n L, n \in \mathbb{N}$, where $r(l)=r_{\min }$. However, it will occur that $\tilde{\mathcal{S}}$ runs through the black hole region and through the past cosmological horizon (or vice versa). This can be seen from the combination of Figs. 10(a) and 10(b)

The embedding of a compact CMC-initial data set $\left(\Sigma,{ }^{3} g_{i j}, K_{i j}\right), \Sigma \cong S^{1} \times S^{2}$, as a CMChypersurface $\mathcal{S}$ in KSSdS[T] is more delicate.

Consider (24) and make the following

\section{Definition.}

$$
\mathcal{T}:=t(L)=\int_{0}^{L} V^{-1}(r(l))\left(\frac{K r(l)}{3}-\frac{C}{r(l)^{2}}\right) d l=\int_{r_{\min }}^{r_{\max }} V^{-1} D^{-1 / 2}(r)\left(\frac{K r}{3}-\frac{C}{r^{2}}\right) d r .
$$

Like $r_{\text {min }}$ and $r_{\text {max }}$, the function $\mathcal{T}$ depends on the pair $(K, C) \in \mathcal{K} \mathcal{C}_{0}$ characterizing the CMC-data. For $(K, C) \in C_{b, t}, \mathcal{T}$ is not canonically defined; each choice of $l_{0}, l_{1}$, which leads to compact data, cf. Sec. 2] is associated with a different value of $\mathcal{T}$.

Proposition 3.1. Let $(K, C) \in \mathcal{K C}_{0}$. Then the associated compact CMC-initial data set $\left(\Sigma \cong S^{1} \times S^{2},{ }^{3} g_{i j}, K_{i j}\right)$ is embeddable as a CMC-hypersurface $\mathcal{S}$ in $K S S d S[T]$ if and only if $T=\mathcal{T}$. The hypersurface is a Cauchy hypersurface, and the embedding is unique modulo the Killing flow.

Proof. Consider the universal covering $\tilde{\Sigma}$ of the data and the embedded hypersurface $\tilde{\mathcal{S}}$ as given by (24). The hypersurface is invariant under the two discrete isometries $t \mapsto-t$ and 
$2 \mathcal{T}+t \mapsto 2 \mathcal{T}-t$ of the spacetime; this is because $r(l)$ is even about $l=0$ and even about $l=2 L$ according to its periodicity. Since the unit normal vectors of $\tilde{\mathcal{S}}$ at $l=0$ and $l=2 L$ must be invariant under the respective isometries as well, they must be tangential to the fixed point sets $t=0$ and $t=2 \mathcal{T}$ of the isometries: we conclude that $n^{\mu} \partial_{\mu} \propto \partial_{t}$ at $l=0$ and $l=2 L$. The identification of $t=0$ with $t=2 \mathcal{T}$ in KSSdS thus results in a CMC-hypersurface $\mathcal{S}$ in $\operatorname{KSSdS}[\mathcal{T}]$ which is isometric to $\Sigma$ by construction. The proof of the remaining claims is trivial.

The argument used in the proof will re-appear in the proof of Theorem 4.1] alternatively, we could have used (25).

Remark. Consider (the universal covering of) the CMC-initial data generated by $(K, C) \in$ $C_{b, t} \subset \partial(\mathcal{K C})$. Since the data is characterized by a function $r(l) \equiv$ const, the embedding is a $r=$ const hypersurface, which is contained in the black/white hole region of KSSdS[T]

(for arbitrary $\mathrm{T}$ ). The compact interpretation of the data set, which is obtained by an identification of $l=l_{0}$ with $l=l_{1}$ for any $l_{0}, l_{1}$, is not naturally embeddable in KSSdS[T].

\section{Slicings}

Proposition 3.1 states that there exist spacetimes KSSdS[T] containing a compact CMChypersurface. The aim of this section is to investigate whether these spacetimes contain $C M C$-slicings, i.e. smooth families of CMC-hypersurfaces.

Any CMC-hypersurface can be evolved into a CMC-slicing by the Killing flow; however, this CMC-slicing is trivial in the sense that $K$ and $C$ are constant along the slicing. The existence of non-trivial slicings is shown in the following theorem: any compact CMC-hypersurface of $\mathrm{KSSdS}[\mathrm{T}]$ evolves into a compact CMC-slicing, along which the mean curvature $K$ is monotonic; furthermore, this slicing is necessarily unique modulo the Killing flow.

Theorem 4.1. Consider a spacetime KSSdS[T] that contains a compact CMC-hypersurface $\mathcal{S}$. Then there exists a unique local slicing of KSSdS[T] by hypersurfaces $\mathcal{S}_{\tau}, \tau \in(-\bar{\tau}, \bar{\tau})$, such that

i. $\mathcal{S}_{0}=\mathcal{S}$

ii. $\mathcal{S}_{\tau}$ is a compact CMC-hypersurface for all $\tau \in(-\bar{\tau}, \bar{\tau})$,

iii. $\mathcal{S}_{\tau}$ is reflection symmetric for all $\tau$.

Along the slicing $\mathcal{S}_{\tau}$ the mean curvature is a strictly monotonic function, i.e.

iv. $K(\tau)$ is strictly monotonic.

Furthermore, every slicing $\mathcal{S}_{\tau}^{\prime}$ that satisfies (2) and (2i]) arises from $\mathcal{S}_{\tau}$ by combining the flow of $\mathcal{S}_{\tau}$ with an appropriate admixture of the Killing flow.

Remark. The requirement (iii) is a convenient way of fixing a representative within the equivalence class of slicings that satisfy (ii) and (iii). The compact CMC-hypersurface $\mathcal{S}$ contains a totally geodesic 2-sphere at $r=r_{\min }$, which evolves into a cylinder represented by $\tau \mapsto r_{\min }(\tau)$. 
The slicing $\mathcal{S}_{\tau}$ is reflection symmetric if the cylinder is totally geodesic, i.e. coincides with $t=$ const; without loss of generality we always assume reflection symmetry w.r.t. $t=0$.

Proof. The CMC-hypersurface $\mathcal{S}$ is characterized by $\left(K_{\mathcal{S}}, C_{\mathcal{S}}\right) \in \mathcal{K C}_{0}$; the induced metric is $d l^{2}+r_{\mathcal{S}}^{2}(l) d \Omega^{2}$. $\mathcal{S}$ can be represented by (24), it is invariant under the reflection $t \mapsto-t$. We introduce Gaussian coordinates in a neighborhood of $\mathcal{S}$ : the metric reads

$$
g_{\mu \nu} d x^{\mu} d x^{\nu}=-d \sigma^{2}+s^{2}(\sigma, \lambda) d \lambda^{2}+r^{2}(\sigma, \lambda) d \Omega^{2}
$$

$\sigma \in(-\bar{\sigma}, \bar{\sigma})$. The hypersurface $\mathcal{S}$ is represented by $\sigma=0 ; \lambda=l$ on $\mathcal{S}$. We obtain by using the identity $\partial_{\sigma} g_{i j}=2 K_{i j}$ and ([6]),

$$
s(0, \lambda)=1, \quad r(0, \lambda)=r_{\mathcal{S}}(\lambda), \quad\left(\partial_{\sigma} s\right)(0, \lambda)=\frac{K_{\mathcal{S}}}{3}+\frac{2 C_{\mathcal{S}}}{r_{\mathcal{S}}^{3}(\lambda)}, \quad\left(\partial_{\sigma} r\right)(0, \lambda)=\frac{K_{\mathcal{S}} r_{\mathcal{S}}(\lambda)}{3}-\frac{C_{\mathcal{S}}}{r_{\mathcal{S}}^{2}(\lambda)} .
$$

We choose $\bar{\sigma}$ so that $\inf _{|\sigma|<\bar{\sigma}} s(\sigma, \lambda) \geq$ const and $\inf _{|\sigma|<\bar{\sigma}} r(\sigma, \lambda) \geq$ const $\forall \lambda$. The normal vector of $\mathcal{S}$ at $\lambda=0$ is tangential to the cylinder $t=0$, since $\mathcal{S}$ is invariant under the discrete isometry $t \mapsto-t$. The cylinder $t=0$, being the fixed point set of a discrete isometry, is totally geodesic, therefore the normal geodesics passing through $\lambda=0$ lie on $t=0$, hence the hypersurfaces $\lambda=0$ and $t=0$ coincide; analogously, $\lambda=L_{\mathcal{S}}$ corresponds to $t=\mathcal{T}_{\mathcal{S}}(=\mathrm{T})$. From this fact that the Gaussian coordinates are adapted to the discrete symmetry, it thus follows that $s(\sigma, \lambda)$ and $r(\sigma, \lambda)$ are even functions in $\lambda$ for all $\sigma$; analogously, the functions are even about $\lambda=L_{\mathcal{S}}$.

In a neighborhood of $\mathcal{S}$ in $\mathrm{KSSdS}[\mathrm{T}]$, a compact hypersurface can be described by the equations

$$
\sigma=\varphi(l), \quad \lambda=l, \quad \text { where } \quad \varphi: S^{1} \rightarrow(-\bar{\sigma}, \bar{\sigma}) .
$$

We define the mean curvature operator $\mathcal{K}$,

$$
\mathcal{K}[\varphi]:=\frac{1}{\sqrt{s^{2}-\varphi^{\prime 2}}}\left(\partial_{\sigma} s+\frac{2 s \partial_{\sigma} r}{r}\right)-\frac{\varphi^{\prime}}{\left(s^{2}-\varphi^{\prime 2}\right)^{3 / 2}}+\frac{2 r^{\prime}}{r s} \frac{\varphi^{\prime}}{\sqrt{s^{2}-\varphi^{\prime 2}}}+\frac{1}{s}\left(\frac{\varphi^{\prime}}{\sqrt{s^{2}-\varphi^{\prime 2}}}\right)^{\prime} ;
$$

for $\varphi$, the prime denotes a derivative w.r.t. $l$, for $s$ and $r$, a derivative w.r.t. the second argument; the suppressed arguments of the functions $s, r$, and its derivatives, are $(\varphi(l), l)$. For a given $\varphi, \mathcal{K}[\varphi]$ is a function $S^{1} \rightarrow \mathbb{R}$ describing the mean curvature of the hypersurface $\sigma-\varphi(\lambda)=0$. The hypersurface $\mathcal{S}$ is represented by $\varphi=0$; from (28) we obtain $\mathcal{K}[0]=K_{\mathcal{S}}$. In order to show the claims of the theorem we solve the prescribed mean curvature equation $\mathcal{K}[\varphi]=K$ (三 const) in a neighborhood of $\mathcal{S}$.

Consider the Sobolev space $H^{2}\left(S^{1}\right)=W^{2,2}\left(S^{1}\right)$. The Sobolev inequalities ensure that $\psi \in$ $H^{2}\left(S^{1}\right)$ implies $\psi \in \mathcal{C}^{1,1 / 2}\left(S^{1}\right)$, where $\mathcal{C}^{1,1 / 2}$ denotes the relevant Hölder space; we have the estimate $\|\psi\|_{\mathcal{C}^{1,1 / 2}} \leq$ const $\|\psi\|_{H^{2}}$ for all $\psi$. Since $\|\psi\|_{L^{\infty}}+\left\|\psi^{\prime}\right\|_{L^{\infty}} \leq\|\psi\|_{\mathcal{C}^{1,1 / 2}}$, the set ${ }^{\delta} H^{2}\left(S^{1}\right):=\left\{\psi \in H^{2}\left(S^{1}\right) \mid\|\psi\|_{L^{\infty}}+\left\|\psi^{\prime}\right\|_{L^{\infty}}<\delta=\mathrm{const}\right\}$ is a neighborhood of the origin in $H^{2}\left(S^{1}\right)$. Let $H_{\text {even }}^{k}\left(S^{1}\right)$ denote the space of even functions $\psi \in H^{k}\left(S^{1}\right)$; as a closed subspace of $H^{k}\left(S^{1}\right)$ it is a Banach space. Finally define ${ }^{\delta} H_{\text {even }}^{2}\left(S^{1}\right):=H_{\text {even }}^{2}\left(S^{1}\right) \cap{ }^{\delta} H^{2}\left(S^{1}\right)$. We choose $\delta$ sufficiently small, so that $\|\psi\|_{L^{\infty}}<\bar{\sigma}$ and $\left\|s^{2}-\psi^{\prime 2}\right\|_{L^{\infty}} \geq$ const $>0$. The quasilinear operator $\mathcal{K}$, viewed as a map

$$
\mathcal{K}:{ }^{\delta} H_{\text {even }}^{2}\left(S^{1}\right) \rightarrow H_{\text {even }}^{0}\left(S^{1}\right) .
$$


is $C^{1}$-differentiable. The argument is standard and can be inferred, e.g. from Thm. 4.1 in [11]. The (Fréchet) derivative of $\mathcal{K}$ at a point $\varphi$ is a linear operator,

$$
\mathcal{K}^{\prime}[\varphi]: H_{\text {even }}^{2}\left(S^{1}\right) \rightarrow H_{\text {even }}^{0}\left(S^{1}\right),
$$

which, for $\varphi$ regular enough, is given by

$$
\mathcal{K}^{\prime}[\varphi](\dot{\varphi})=\left(\Delta_{\left({ }^{3} g_{\varphi}\right)}+\Lambda-K_{\varphi i j} K_{\varphi}^{i j}\right)\left(\frac{s}{\sqrt{s^{2}-\varphi^{\prime 2}}} \dot{\varphi}\right)
$$

with $\dot{\varphi} \in H_{\text {even }}^{2}\left(S^{1}\right)$. The expressions ${ }^{3} g_{\varphi}$ and $K_{\varphi}$ are the metric and extrinsic curvature of the surface $\sigma-\varphi(\lambda)=0$; the Laplacian is the one associated with ${ }^{3} g_{\varphi}$. It is convenient to obtain Eq. (33) through geometric arguments, cf. the remark following Corollary 4.3

Since $\varphi \equiv 0$ represents the CMC-hypersurface $\mathcal{S}$ we obtain

$$
\mathcal{K}^{\prime}[0]=\Delta+a \quad \text { where } \quad a(l)=\Lambda-\frac{K_{\mathcal{S}}^{2}}{3}-\frac{6 C_{\mathcal{S}}^{2}}{r^{6}(l)} .
$$

(Note that there do not exist pairs $\left(K_{\mathcal{S}}, C_{\mathcal{S}}\right) \in \mathcal{K C}_{0}$ such that $a(l) \leq 0 \forall l$.) The operator $\mathcal{K}^{\prime}[0]$ is elliptic, the Fredholm alternative holds. In Lemma 4.2 we show that ker $\mathcal{K}^{\prime}[0]$ is trivial, i.e. the homogeneous equation $\Delta \alpha+a \alpha=0$ admits only the trivial solution $\alpha=0$. It follows that $\mathcal{K}^{\prime}[0]$ is an isomorphism, and we are able to apply the inverse function theorem, see, e.g. [8]: there exists an open neighborhood $V$ of $\mathcal{K}[0]$ in $H_{\text {even }}^{0}\left(S^{1}\right)$ and a unique continuously differentiable mapping $\mathcal{K}^{-1}: V \rightarrow{ }^{\delta} H_{\text {even }}^{2}\left(S^{1}\right)$ with the property that $\mathcal{K}\left[\mathcal{K}^{-1}(\kappa)\right]=\kappa$ for all $\kappa \in V$.

Let $K$ be a smooth real function of $\tau \in \mathbb{R}$ which is strictly monotonic and satisfies $K(0)=K_{\mathcal{S}}$; let $(-\bar{\tau}, \bar{\tau})$ be an interval such that $K(\tau) \in V$ for all $\tau \in(-\bar{\tau}, \bar{\tau})$; note that $K(\tau)$ is interpreted as a constant function for each $\tau$. Then

$$
\varphi_{\tau}:=\mathcal{K}^{-1}(K(\tau))
$$

uniquely defines a family of ${ }^{\delta} H_{\text {even }}^{2}\left(S^{1}\right)$-functions $\left\{\varphi_{\tau} \mid \tau \in(-\bar{\tau}, \bar{\tau})\right\}$, where $\varphi_{\tau_{1}} \neq \varphi_{\tau_{2}}$ for $\tau_{1} \neq \tau_{2}$. For each $\tau, \varphi_{\tau}$ is smooth, by elliptic regularity. Moreover, the mapping $\tau \rightarrow \varphi_{\tau}$ is continuously differentiable by construction. Therefore $\left\{\varphi_{\tau}\right\}$ defines a unique local slicing of KSSdS[T] by compact CMC-hypersurfaces

$$
\mathcal{S}_{\tau}:=\left\{(\sigma, \lambda, \Omega) \in \mathrm{KSSdS}[\mathrm{T}] \mid \sigma=\varphi_{\tau}(l), \lambda=l\right\},
$$

$\tau \in(-\bar{\tau}, \bar{\tau}) ; \mathcal{S}_{\tau_{1}} \neq \mathcal{S}_{\tau_{2}}$ for $\tau_{1} \neq \tau_{2} ;$ since $\varphi_{0} \equiv 0, \mathcal{S}_{0}=\mathcal{S}$. By construction, since $\varphi_{\tau}$ is even for each $\tau$, and since $\lambda=0$ coincides with $t=0, \mathcal{S}_{\tau}$ is invariant under the discrete isometry $t \mapsto-t$ for all $\tau$. Hence, the properties (ii)-(iii) are proved; also (iv) is a direct consequence of the construction. The remaining claim follows immediately from the considerations of Sec. 2 and Prop. 3.1

Remark. In the proof, $K(\tau)$ is strictly monotonic, which does not imply that $\dot{K} \neq 0$ a priori. However, by an appropriate redefinition of the parameter $\tau, \dot{K} \neq 0$ can always be achieved. Henceforth we will always adopt the convention that $\dot{K}>0$. 
Lemma 4.2. Consider the equation

$$
\Delta \alpha+a \alpha=0
$$

where $a(l)=\Lambda-K^{2} / 3-6 C^{2} / r^{6}(l)$, and let $\alpha \in H_{\mathrm{even}}^{2}\left(S^{1}\right)$ be a solution. Then $\alpha \equiv 0$.

For the proof we refer to Appendix $[$ ]

Corollary 4.3. The equation

$$
\Delta \alpha+a \alpha=\dot{K} \quad(\dot{K}=\text { const }>0)
$$

possesses a unique even solution $\alpha: S^{1} \rightarrow \mathbb{R}$ for all $(K, C) \in \mathcal{K C}_{0}$.

Proof. The equation is elliptic, the homogeneous equation possesses only the trivial solution. The Fredholm alternative holds, hence existence and uniqueness of an even periodic solution $\alpha$ follows.

Equation (38) is obtained by differentiating the mean curvature ${ }^{3} g^{i j} K_{i j}$ and using the evolution equations along the slicing. The solution of (38) is the lapse function of the slicing $\mathcal{S}_{\tau}$ described in Theorem 4.1] in fact, when we set

$$
\Phi:(\tau, l, \Omega) \mapsto(\sigma, \lambda, \Omega)=\Phi(\tau, l, \Omega)=(\varphi(\tau, l), l, \Omega),
$$

where $\varphi(\tau, \cdot)=\varphi_{\tau}(\cdot)$, and note that the future unit (co)normal of the slicing $\mathcal{S}_{\tau}$ is given by

$$
n_{\mu} d x^{\mu}=\frac{s}{\sqrt{s^{2}-\varphi^{\prime 2}}}\left(-d \sigma+\varphi^{\prime} d \lambda\right)
$$

we obtain

$$
\alpha=\frac{s}{\sqrt{s^{2}-\varphi^{\prime 2}}} \dot{\varphi}
$$

from the decomposition $\dot{\Phi}^{\mu}=\alpha n^{\mu}+\Phi_{, i}^{\mu} X^{i}$ (where the dot denotes $\partial / \partial \tau$ ). Hence (33) and (38) coincide.

The shift vector of the slicing $\mathcal{S}_{\tau}$ depends on the "spatial gauge" we are imposing. Let us briefly elaborate on this issue. Consider the parametrization of the slicing

$$
\Phi:(\tau, l, \Omega) \mapsto(\sigma, \lambda, \Omega)=\Phi(\tau, l, \Omega)=\left(\varphi\left(\tau, \beta_{\tau}(l)\right), \beta_{\tau}(l), \Omega\right),
$$

where $\beta_{\tau}(\cdot):\left[-L_{\mathcal{S}}, L_{\mathcal{S}}\right]_{\sim} \cong S^{1} \rightarrow S^{1}$ is one-to-one for each $\tau$; when the parametrization is adapted to the reflection symmetry of the slicing, $\beta_{\tau}(0)=0$ must hold; by virtue of the $S^{1}$-periodicity, $\beta_{\tau}\left( \pm L_{\mathcal{S}}\right)= \pm L_{\mathcal{S}}$ for all $\tau$. On each hypersurface $\mathcal{S}_{\tau}, l$ is a coordinate, however, the 3-metric on $\mathcal{S}_{\tau}$ is not of the form $d l^{2}+r^{2} d \Omega^{2}$ for general $\beta_{\tau}$. Now consider $\dot{\Phi}^{\mu} \partial_{\mu}=\left(\dot{\varphi}+\dot{\beta} \varphi^{\prime}\right) \partial_{\sigma}+\dot{\beta} \partial_{\lambda}$ and $\Phi_{, l}^{\mu} \partial_{\mu}=\varphi^{\prime} \beta^{\prime} \partial_{\sigma}+\beta^{\prime} \partial_{\lambda}$, where $\beta(\tau, \cdot)=\beta_{\tau}(\cdot)$. While the lapse $\alpha$ is given by (41), the shift vector field $X^{i} \partial_{i}=x \partial_{l}, x=x(\tau, l)$, depends on the gauge $\beta_{\tau}$. It is given explicitly by

$$
x=-\frac{1}{\beta^{\prime}} \frac{\varphi^{\prime} \dot{\varphi}}{s^{2}-\varphi^{\prime 2}}+\frac{\dot{\beta}}{\beta^{\prime}}=-\frac{\varphi^{\prime}}{\beta^{\prime}} \frac{\alpha}{s \sqrt{s^{2}-\varphi^{\prime 2}}}+\frac{\dot{\beta}}{\beta^{\prime}},
$$


where we again suppress the arguments $(\tau, \beta(\tau, l))$ on the r.h. side. Note that $x(\tau, 0)=0$ and $x\left(\tau, \pm L_{\mathcal{S}}\right)=0$, since $\varphi^{\prime}\left(\tau, \pm L_{\mathcal{S}}\right)=0$ and $\beta\left(\tau, \pm L_{\mathcal{S}}\right)=0$. We mention three gauges: when $\beta_{\tau}(l)=l$ we recover the gauge used in Theorem 4.1, cf. (29), and $x=-\alpha s^{-1}\left(s^{2}-\varphi^{\prime 2}\right)^{-1 / 2} \varphi^{\prime}$. Another interesting parametrization is the one given by $\dot{\beta}=\left[s^{2}-\varphi^{\prime 2}\right]^{-1} \varphi^{\prime} \dot{\varphi}$; this gives vanishing shift, i.e. an "Eulerian" gauge. As a third possibility, the gauge given by $\beta^{\prime}=$ $\left[s^{2}-\varphi^{\prime 2}\right]^{-1 / 2}$ suggests itself. Here the spatial metric reads $d l^{2}+r^{2} d \Omega$ for all $\tau$, as is easily seen by considering the pull-back of (27); we call this the "isotropic gauge". The shift, which reads $x=-\alpha \varphi^{\prime}+\sqrt{s^{2}-\varphi^{\prime 2}} \dot{\beta}$, satisfies $x^{\prime}=-\alpha\left(K / 3-2 C / r^{3}\right)$, as can be shown by using (28) or by invoking the identity $\partial_{\tau}\left(\Phi^{\mu}{ }_{i} \Phi^{\nu}{ }_{j} g_{\mu \nu}\right)=2 \alpha K_{i j}+2 D_{(i} X_{j)}$. But beware: the isotropic gauge is not consistent with the $S^{1}$-periodicity requirement, and thus $x\left(\tau, \pm L_{\mathcal{S}}\right) \neq 0$.

The following relations are recorded for later use. Consider $r=r(\Phi(\tau, l))$ and $t=t(\Phi(\tau, l))$ in any gauge. Restricted to $\mathcal{S}$ (i.e. for $\tau=0$ or $\sigma=0$ ), we have

$$
\frac{\partial r}{\partial \tau}=\left(\frac{K_{\mathcal{S}} r}{3}-\frac{C_{\mathcal{S}}}{r^{2}}\right) \alpha+x r^{\prime}, \quad \frac{\partial t}{\partial \tau}=V^{-1}\left[r^{\prime} \alpha+\left(\frac{K_{\mathcal{S}} r}{3}-\frac{C_{\mathcal{S}}}{r^{2}}\right) x\right] .
$$

To prove the first relation we compute

$$
\left.\dot{r}\right|_{\tau=0}=\left.\left.\left(\partial_{\sigma} r\right)\right|_{\sigma=0}\left(\dot{\varphi}+\varphi^{\prime} \dot{\beta}\right)\right|_{\tau=0}+\left.\left.\left(\partial_{\lambda} r\right)\right|_{\sigma=0} \dot{\beta}\right|_{\tau=0}=\left[\left(\frac{K_{\mathcal{S}} r}{3}-\frac{C_{\mathcal{S}}}{r^{2}}\right) \alpha+r^{\prime} x\right]_{\tau=0}
$$

where we have used (28) and the fact that $\varphi(0, l) \equiv 0$ and $\beta(0, l) \equiv l$. To show the second relation we note that the Killing vector is given by $\xi=r^{\prime} \partial_{\sigma}-\left[K_{\mathcal{S}} r / 3-C_{\mathcal{S}} / r^{2}\right] \partial_{\lambda}$ for $\sigma=0$, cf. (12). Hence for $\xi_{\nu} d x^{\nu}$ we obtain $\xi_{\sigma}=-r^{\prime}$ and $\xi_{\lambda}=-\left[K_{\mathcal{S}} r / 3-C_{\mathcal{S}} / r^{2}\right]$, both evaluated at $\sigma=0$, cf. (27). Now, $\partial_{\nu} t=-V^{-1} \xi_{\nu}$, cf. (17), therefore

$$
\left.\dot{t}\right|_{\tau=0}=\left.\left.\left(\partial_{\sigma} t\right)\right|_{\sigma=0} \alpha\right|_{\tau=0}+\left.\left.\left(\partial_{\lambda} t\right)\right|_{\sigma=0} x\right|_{\tau=0}=V^{-1}\left[r^{\prime} \alpha+\left(\frac{K_{\mathcal{S}} r}{3}-\frac{C_{\mathcal{S}}}{r^{2}}\right) x\right]_{\tau=0} .
$$

Proposition 4.4. Consider a spacetime KSSdS[T] that contains a CMC-slicing $\mathcal{S}_{\tau}, \tau \in$ $(-\bar{\tau}, \bar{\tau})$, satisfying the properties (i)-(iii) of Theorem [4.1. This slicing uniquely corresponds to a smooth curve in $\mathcal{K C}_{0}$,

$$
(-\bar{\tau}, \bar{\tau}) \ni \tau \mapsto(K, C)(\tau) \in \mathcal{K} \bigodot_{0},
$$

such that $(K, C)(0)=\left(K_{\mathcal{S}}, C_{\mathcal{S}}\right)$. The tangent vector $(\dot{K}, \dot{C})(\tau)$ of the curve is given through

$$
-\frac{\dot{K}(\tau) r_{\min }(\tau)}{3}+\frac{\dot{C}(\tau)}{r_{\min }^{2}(\tau)}=r_{\min }^{\prime \prime}(\tau) \alpha_{\min }(\tau),
$$

where $r_{\min }(\tau)=r(\tau ; 0), r_{\min }^{\prime \prime}(\tau)=r^{\prime \prime}(\tau ; 0)=\left.(1 / 2)(d D(\tau ; r) / d r)\right|_{r=r_{\min }(\tau)}, \alpha_{\min }(\tau)=\alpha(\tau ; 0)$; $\alpha(\tau ; l)$ is the lapse function of the slicing.

Proof. $\mathcal{S}_{\tau}$ is a compact CMC-hypersurface in $\mathrm{KSSdS}[\mathrm{T}]$ for all $\tau$, hence the CMC-data is characterized by $(K(\tau)), C(\tau)) \in \mathcal{K C}_{0}$ for all $\tau$. Accordingly, $\mathcal{S}_{\tau}$ generates a mapping $(-\bar{\tau}, \bar{\tau}) \ni$ $\tau \mapsto(K, C)(\tau)=(K(\tau), C(\tau)) \in \mathcal{K C}_{0}$; clearly, $(K, C)(0)=(K(0), C(0))=\left(K_{\mathcal{S}}, C_{\mathcal{S}}\right)$. Вy virtue of the uniqueness property of $\mathcal{S}_{\tau}$ established in Theorem 4.11, the connection between 
the slicing $\mathcal{S}_{\tau}$ and the map $(K, C)(\tau)$ is one-to-one. To prove that $(K, C)(\tau)$ is a smooth curve in $\mathcal{K C}_{0}$, consider

$$
K_{i j}(\tau ; r) K^{i j}(\tau ; r)=\frac{K^{2}(\tau)}{3}+\frac{6 C^{2}(\tau)}{r^{6}},
$$

cf. (6). Along the (smooth) slicing $\mathcal{S}_{\tau}, K_{i j} K^{i j}$ and $K$ are smooth functions, therefore $C(\tau)$ and thus $(K, C)(\tau)$ is smooth.

Let $\alpha(\tau ; l)$ be the lapse function of the slicing $\mathcal{S}_{\tau}$ and $X^{i} \partial_{i}=x(\tau ; l) \partial_{l}$ the shift vector in an arbitrary gauge. Consider $\partial_{\tau}\left[K_{i j}(\tau ; l) K^{i j}(\tau ; l)\right]$. We have on one hand that

$$
\left(K_{i j} K^{i j}\right)^{\cdot}=\frac{2}{3} K \dot{K}+\frac{12 C}{r^{6}} \dot{C}-\frac{36 C^{2}}{r^{7}} \dot{r},
$$

where $\dot{r}$ is given by (44). On the other hand, from the evolution equations and by employing (38),

$$
\left(K_{i j} K^{i j}\right)^{\cdot}=-\frac{12 C \alpha}{r^{6}}(-r D(r)+r-3 M)-\frac{12 C r^{\prime} \alpha^{\prime}}{r^{4}}+2\left(\frac{K}{3}+\frac{2 C}{r^{3}}\right) \dot{K}-\frac{36 C^{2}}{r^{7}} r^{\prime} x .
$$

Equating (50) and (51), the terms involving the shift cancel, and we obtain

$$
r^{\prime}(\tau ; l) \alpha^{\prime}(\tau ; l)-r^{\prime \prime}(\tau ; l) \alpha(\tau ; l)=\frac{\dot{K}(\tau) r(\tau ; l)}{3}-\frac{\dot{C}(\tau)}{r^{2}(\tau ; l)},
$$

from which Eq. (48) ensues by evaluation at $l=0$.

Definition. Equation (48), i.e.

$$
-\frac{\dot{K} r_{\min }}{3}+\frac{\dot{C}}{r_{\min }^{2}}=r_{\min }^{\prime \prime} \alpha_{\min },
$$

together with $\dot{K}>0$, defines a unique oriented direction field on $\mathcal{K C}_{0}$. This is because $r_{\text {min }}$, $r_{\min }^{\prime \prime}$, and $\alpha_{\min }$ can be regarded as functions of $(K, C) \in \mathcal{K C}_{0}$. (Note in particular that the lapse function is determined by equation (38), which only relies on the initial data sets.)

Proposition 4.4 states that a slicing $\mathcal{S}_{\tau}$ is uniquely represented by a (local) integral curve of the direction field. The following proposition turns this into a global statement:

Proposition 4.5. Consider a spacetime KSSdS[T] that contains a CMC-slicing $\mathcal{S}_{\tau}, \tau \in$ $(-\bar{\tau}, \bar{\tau})$, satisfying the properties (지)-(iii) of Theorem 4.1. This slicing can be maximally extended to a slicing $\mathcal{S}_{\tau}, \tau \in\left(\tau_{-}, \tau_{+}\right) \supseteq(-\bar{\tau}, \bar{\tau})$, satisfying (지)-(iii), such that

$$
(K, C)(\tau) \rightarrow \partial\left(\mathcal{K} \bigodot_{0}\right) \quad\left(\tau \rightarrow \tau_{ \pm}\right)
$$

Thus, the maximal extension of a slicing uniquely corresponds to a maximal integral curve of the oriented direction field in $\mathcal{K C}_{0}$. 
Proof. Let $\left(\tau_{-}, \tau_{+}\right)$be the maximal interval of existence of the slicing $\mathcal{S}_{\tau}$ provided by Theorem 4.1, and consider the associated curve $(K, C)(\tau)$ in $\mathcal{K C}_{0}$, which is an open piece of a maximal integral curve $(K, C)_{\text {mic }}(\tau)$ of the direction field defined on $\mathcal{K C}_{0}$. Assume that $(K, C)(\tau) \rightarrow\left(K_{+}, C_{+}\right) \in \mathcal{K}_{0}$ as $\tau \rightarrow \tau_{+}$. Since $\mathcal{S}_{\tau}$ is a hypersurface in $\operatorname{KSSdS}[\mathrm{T}], \mathcal{T}(\tau)=\mathrm{T}$ for all $\tau \in\left(\tau_{-}, \tau_{+}\right)$. As a function of $K$ and $C$ on $\mathcal{K} \bigodot_{0}, \mathcal{T}$ is continuous, cf. (26); it follows that $\mathcal{T}\left(K_{+}, C_{+}\right)=\lim _{\tau \rightarrow \tau_{+}} \mathcal{T}(K(\tau), C(\tau))=\mathrm{T}$. Thus, by Theorem 4.1, there exists a unique local slicing $\hat{\mathcal{S}}_{\pi}, \pi \in(-\bar{\pi}, \bar{\pi})$, in KSSdS[T], such that $\hat{\mathcal{S}}_{0}$ is the CMC-hypersurface characterized by $\left(K_{+}, C_{+}\right)$. The slicing $\hat{\mathcal{S}}_{\pi}$ is represented by an open piece of the maximal integral curve $(K, C)_{\text {mic }}$ passing through $\left(K_{+}, C_{+}\right)$; hence $\mathcal{S}_{\tau} \cup \hat{\mathcal{S}}_{\pi}$ is a slicing of KSSdS[T] that extends $\mathcal{S}_{\tau}$. This is a contradiction to the assumption. The argument for $\tau_{-}$is identical.

\section{Properties of the slicings, and foliations}

In this section we prove that each spacetime $\mathrm{KSSdS}[\mathrm{T}]$ contains a unique (maximally extended) slicing by compact CMC-hypersurfaces, and we show that this slicing is a foliation for a certain range of the time parameter. However, we begin with a discussion of the asymptotic behavior of slicings.

Lemma 5.1. Consider the function

$$
\mathcal{T}(K, C)=\int_{r_{\min }(K, C)}^{r_{\max }(K, C)} V^{-1}(r) D^{-\frac{1}{2}}(K, C ; r)\left(\frac{K r}{3}-\frac{C}{r^{2}}\right) d r
$$

cf. (26)). Let $[0,1) \ni \nu \mapsto(K, C)(\nu) \in \mathcal{K C}_{0}$ be a curve such that $(K, C)(\nu) \rightarrow\left(K_{\partial}, C_{\partial}\right) \in$ $\partial\left(\mathcal{K C}_{0}\right)$ as $\nu \rightarrow 1$. Then

$$
\begin{array}{ll}
\mathcal{T} \rightarrow+\infty & \text { if } \quad\left(K_{\partial}, C_{\partial}\right) \in\left(C_{t} \cup(K=-\sqrt{3 \Lambda})\right) \\
\mathcal{T} \rightarrow-\infty & \text { if } \quad\left(K_{\partial}, C_{\partial}\right) \in\left(C_{b} \cup(K=+\sqrt{3 \Lambda})\right),
\end{array}
$$

where, however, the points $\left(\sqrt{3 \Lambda}, C_{t}(\sqrt{3 \Lambda})\right)$ and $\left(-\sqrt{3 \Lambda}, C_{b}(-\sqrt{3 \Lambda})\right)$ are excluded.

Proof. Let $\left(K_{\partial}, C_{\partial}\right) \in C_{t}$ with $K_{\partial} \in(-\sqrt{3 \Lambda},+\sqrt{3 \Lambda})$. Choose $\epsilon>0$ small, and write

$$
\mathcal{T}(\nu)=\int_{r_{\min }(\nu)}^{r_{\min }(\nu)+\epsilon} \frac{V^{-1}}{\sqrt{D(\nu ; r)}}\left(\frac{K(\nu) r}{3}-\frac{C(\nu)}{r^{2}}\right) d r+\int_{r_{\min }(\nu)+\epsilon}^{r_{\max }(\nu)} \frac{V^{-1}}{\sqrt{D(\nu ; r)}}\left(\frac{K(\nu) r}{3}-\frac{C(\nu)}{r^{2}}\right) d r .
$$

The second integral converges to a constant as $\nu \rightarrow 1$, since $r_{\min }(\nu)$ and $r_{\max }(\nu)$ converge to the values of $r_{\min }$ and $r_{\max }$ at $\left(K_{\partial}, C_{\partial}\right)$, respectively, and the integrand converges uniformly. In $r \in\left(r_{\min }(\nu), r_{\min }(\nu)+\epsilon\right)$ we make the expansion

$$
D(\nu ; r)=D^{\prime}\left(\nu ; r_{\min }\right)\left(r-r_{\min }\right)+\frac{D^{\prime \prime}\left(\nu ; r_{\min }\right)}{2}\left(r-r_{\min }\right)^{2}+O\left(\left(r-r_{\min }\right)^{3}\right),
$$


where $r_{\min }=r_{\min }(\nu)$ and the prime denotes differentiation w.r.t. $r$ in the present context. Then the first integral becomes

$$
\left.\left[V^{-1}\left(r_{\min }\right)\left(\frac{K r_{\min }}{3}-\frac{C}{r_{\min }^{2}}\right)\right]\right|_{\left(K_{\partial}, C_{\partial}\right)} \times\left[-\frac{\sqrt{2} \log \left[D^{\prime}\left(\nu ; r_{\min }\right)\right]}{\sqrt{D^{\prime \prime}\left(\nu ; r_{\min }\right)}}\right]+D_{\epsilon}+O\left(D^{\prime}\left(\nu ; r_{\min }\right)\right) .
$$

The first factor is a positive number, since $K_{\partial} r_{\min } / 3-C_{\partial} / r_{\min }^{2}$ is negative, cf. Fig. 10(a) $D_{\epsilon}$ is a constant. With $\nu \rightarrow 1, D^{\prime}\left(\nu ; r_{\min }(\nu)\right) \rightarrow 0$, cf. Fig. 5(a) the equation $D^{\prime}\left(r_{\min }\right)=0$ is in fact the defining equation for $C_{b, t}$, see Appendix @ Therefore, $\mathcal{T}(\nu)$ diverges like $-\log \left[D^{\prime}\left(\nu ; r_{\min }(\nu)\right)\right]$, i.e. $\mathcal{T} \rightarrow \infty$ as $\nu \rightarrow 1$. Analogously, we are able to prove that $\mathcal{T}=-\infty$ on $C_{b}$ when $K_{\partial} \neq \pm \sqrt{3 \Lambda}$; the different sign results from the different sign of the quantity $\left(K r_{\min } / 3-C / r_{\min }^{2}\right)$; see Fig. 7 .

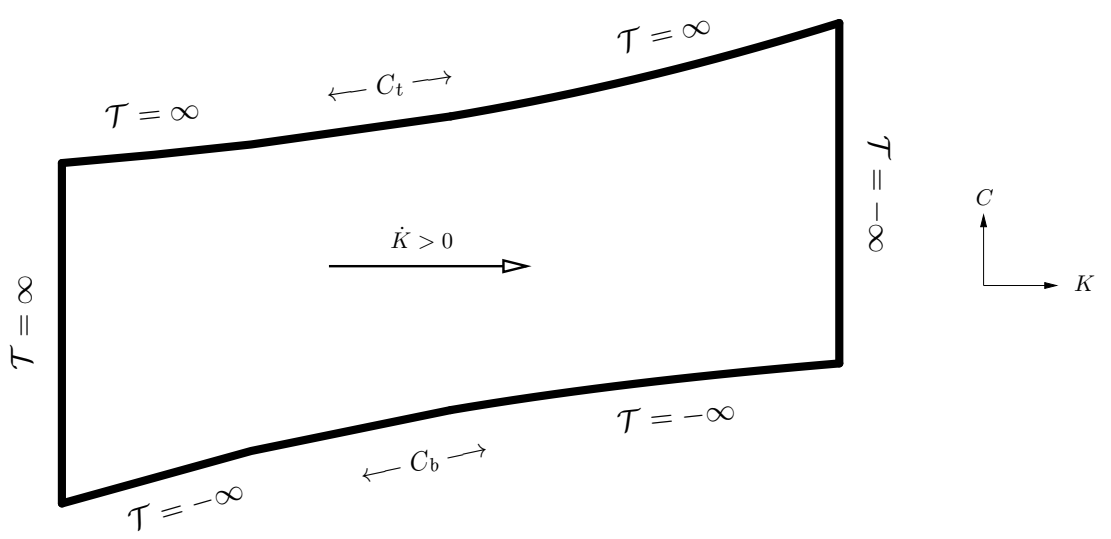

Figure 7: On $\mathcal{K C}_{0}$ the direction field $(\dot{K}, \dot{C})$ satisfies $\dot{K}>0$. On the boundaries $\mathcal{T}= \pm \infty$.

Now let $K_{\partial}= \pm \sqrt{3 \Lambda}, C_{\partial} \in\left(C_{b}( \pm \sqrt{3 \Lambda}), C_{t}( \pm \sqrt{3 \Lambda})\right)$. Along the curve $(K, C)(\nu), r_{\max }(\nu)$ diverges like $\sqrt{3} / \sqrt{\Lambda-K(\nu)^{2} / 3}$, cf. Sec. 2. Choose $r_{0}>r_{\mathrm{c}}$ such that $D(\nu ; r)<4, r-2 M>0$, and $|K(\nu)| r^{3}>6|C(\nu)|$ on $r \in\left[r_{0}, r_{\max }(\nu) / 2\right]$ for all $\nu$ sufficiently close to 1 . Then,

$$
\begin{aligned}
\mathcal{T}-\int_{r_{\min }}^{r_{0}} \frac{V^{-1}}{\sqrt{D}}\left(\frac{K r}{3}-\frac{C}{r^{2}}\right) d r & \lessgtr \int_{r_{0}}^{r_{\max } / 2} \frac{V^{-1}}{\sqrt{D}}\left(\frac{K r}{3}-\frac{C}{r^{2}}\right) d r \\
& \lessgtr \frac{1}{4} \int_{r_{0}}^{r_{\max } / 2}\left(-\frac{\Lambda r^{2}}{3}\right)^{-1}\left(\frac{K r}{3}\right) d r=-\left.\frac{1}{4} \frac{K}{\Lambda} \log r\right|_{r_{0}} ^{r_{\max } / 2}
\end{aligned}
$$

where we have suppressed the dependence on $\nu$. We infer that $\mathcal{T}(\nu)$ diverges at least like $-(\operatorname{sign} K) \log \left(\Lambda-K(\nu)^{2} / 3\right)$ as $\nu \rightarrow 1$; hence $\mathcal{T} \rightarrow-\infty$ when $K_{\partial}=\sqrt{3 \Lambda}$ and $\mathcal{T} \rightarrow \infty$ when $K_{\partial}=-\sqrt{3 \Lambda}$. A combination of the arguments used for (59) and (60) also yields that $\mathcal{T} \rightarrow$ $-\infty$ when $\left(K_{\partial}, C_{\partial}\right)=\left(\sqrt{3 \Lambda}, C_{b}(\sqrt{3 \Lambda})\right)$ and $\mathcal{T} \rightarrow \infty$ when $\left(K_{\partial}, C_{\partial}\right) \rightarrow\left(-\sqrt{3 \Lambda}, C_{t}(-\sqrt{3 \Lambda})\right)$.

Proposition 5.2. Consider a spacetime KSSdS[T] that contains a CMC-slicing satisfying the properties (2i)-(ii2) of Theorem [4.1. Let $\mathcal{S}_{\tau}, \tau \in\left(\tau_{-}, \tau_{+}\right)$, be the maximal extension. Then 


$$
\begin{array}{ll}
(K, C)(\tau) \rightarrow\left(\sqrt{3 \Lambda}, C_{t}(\sqrt{3 \Lambda})\right) & \left(\tau \rightarrow \tau_{+}\right), \\
(K, C)(\tau) \rightarrow\left(-\sqrt{3 \Lambda}, C_{b}(-\sqrt{3 \Lambda})\right) & \left(\tau \rightarrow \tau_{-}\right) .
\end{array}
$$

Hereby, the hypersurfaces $\mathcal{S}_{\tau}$ converge to the asymptotic hypersurfaces $\mathcal{S}_{ \pm}, \mathcal{S}_{+}$in the future, $\mathcal{S}_{-}$in the past,

$$
\mathcal{S}_{ \pm}=\left(r=\frac{1}{\sqrt{\Lambda}}[1-\sqrt{1-3 \sqrt{\Lambda} M}]\right) \cup(r=\infty) \subseteq \overline{\mathrm{KSSdS}[\mathrm{T}]}
$$

as $\tau \rightarrow \tau_{ \pm}$, see Fig. 8

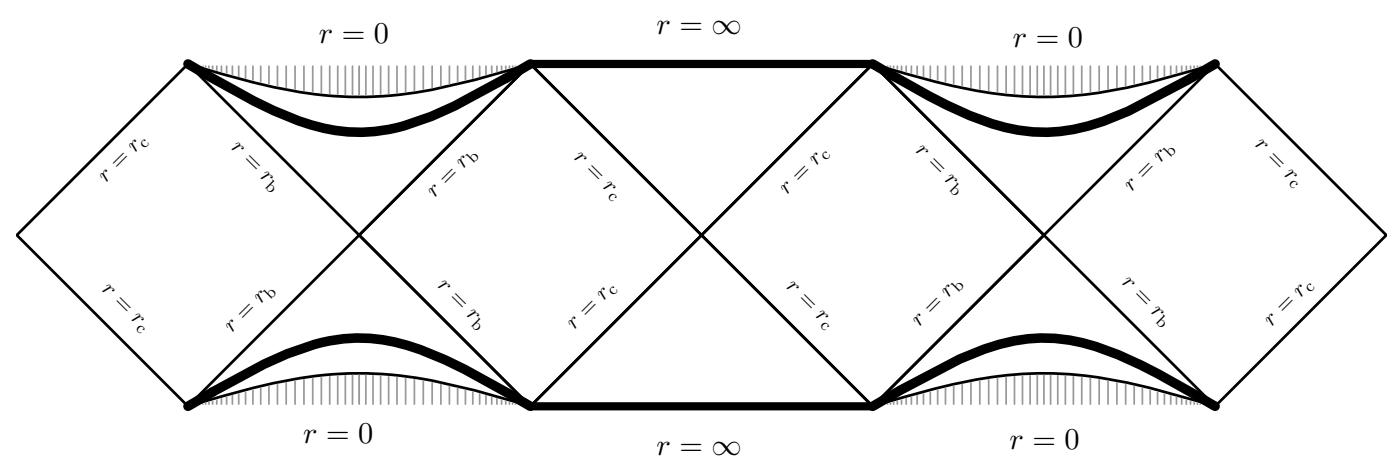

Figure 8: The asymptotic hypersurfaces $\mathcal{S}_{ \pm}$of the slicing $\mathcal{S}_{\tau}$.

Remark. Proposition 5.2 is equivalent to the statement that all maximal integral curves of the oriented direction field on $\mathcal{K}_{0}$ originate from the point $\left(-\sqrt{3 \Lambda}, C_{b}(-\sqrt{3 \Lambda})\right)$ and end in the point $\left(\sqrt{3 \Lambda}, C_{t}(\sqrt{3 \Lambda})\right)$. This is straightforward to prove:

Proof. Each maximal integral curve $(K, C)(\tau)$ of the direction field on $\mathcal{K C}_{0}$ is characterized by $\mathcal{T}(\tau) \equiv \mathrm{T}=$ const, since the slicing is embedded in $\mathrm{KSSdS}[\mathrm{T}]$. Therefore, the limit set of the curve $(K, C)(\tau)$ cannot contain a point on $\partial\left(\mathcal{K} \bigodot_{0}\right)$ where $\mathcal{T}= \pm \infty$, which leaves only the points $\left(-\sqrt{3 \Lambda}, C_{b}(-\sqrt{3 \Lambda})\right)$ and $\left(\sqrt{3 \Lambda}, C_{t}(\sqrt{3 \Lambda})\right)$ by Lemma 5.1. Together with $\dot{K}>0$ this entails (61).

To show the second part of the assertion, we recall that $\mathcal{S}_{\tau}$ can be represented by $t(\tau ; r)$ in KSSdS[T], cf. (24). In analogy to the considerations in the proof of Lemma 5.1 we obtain

$$
t\left(\tau ; r_{\min }(\tau)+\epsilon\right)=\int_{r_{\min }(\tau)}^{r_{\min }(\tau)+\epsilon} \frac{V^{-1}}{\sqrt{D(\tau ; r)}}\left(\frac{K(\tau) r}{3}-\frac{C(\tau)}{r^{2}}\right) d r \rightarrow \infty \quad\left(\tau \rightarrow \tau_{+}\right)
$$

for all $\epsilon>0$; moreover, $r_{\min }(\tau) \rightarrow r_{\min }\left(\tau_{+}\right)$for $\tau \rightarrow \tau_{+}$. Therefore, in the black hole region, $\mathcal{S}_{\tau}$ converges to the hypersurface $r=r_{\min }\left(\tau_{+}\right)$as $\tau \rightarrow \tau_{+}$, where the convergence is uniform on each "cone" $\{t \mid t \in[-E, E], E>0\}$. From (23) we see that $r_{\min }\left(\tau_{+}\right)=(1-\sqrt{1-3 \sqrt{\Lambda} M}) / \sqrt{\Lambda}$. Similar considerations apply for the cosmological region; in particular, $r_{\max }(\tau) \rightarrow \infty$ as $\tau \rightarrow \tau_{+}$. Hence the claim is established. 


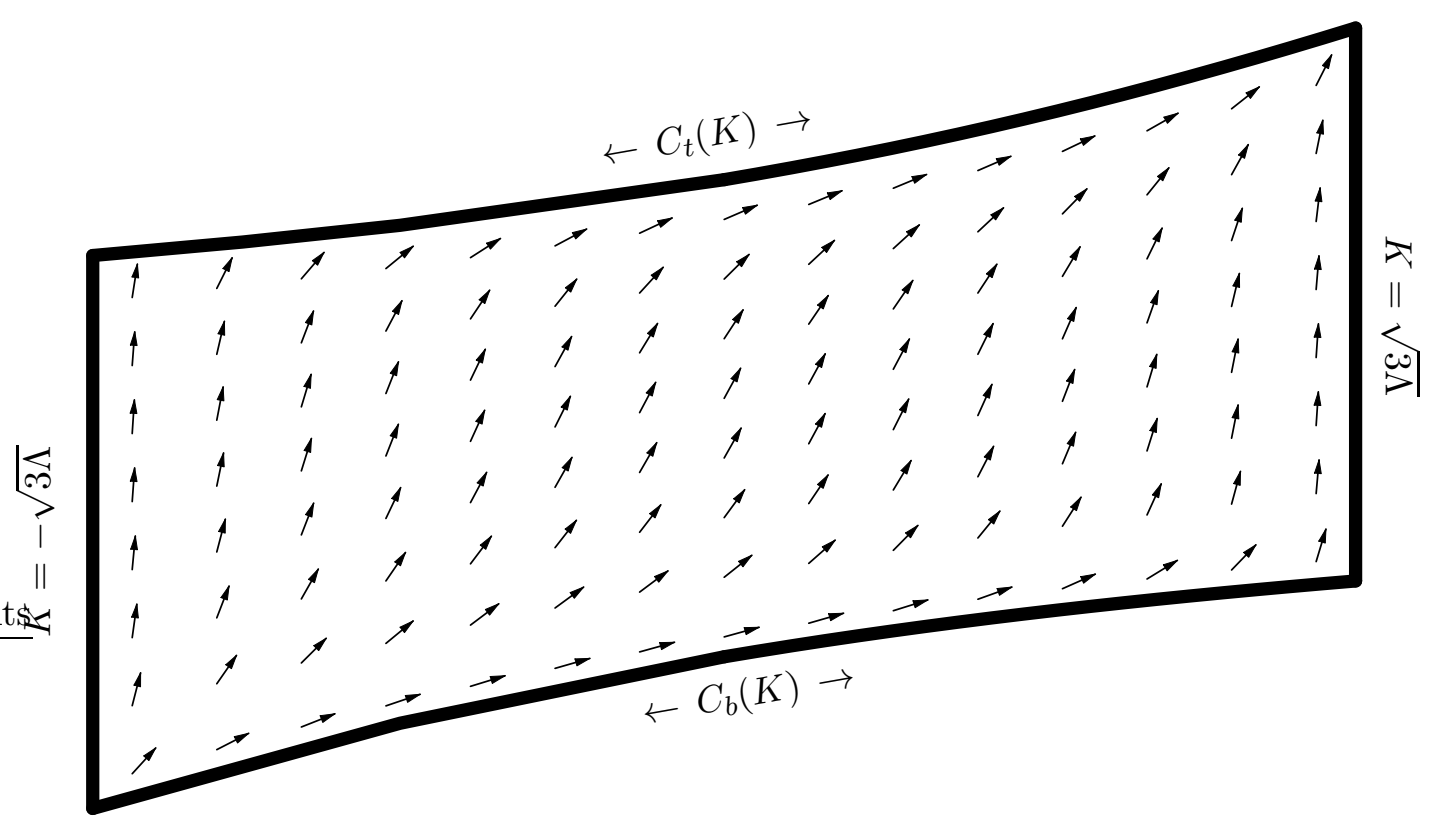

Figure 9: The direction field $(\dot{K}, \dot{C})$ on $\mathcal{K} \mathcal{C}_{0}$ for $\Lambda=1, M=1 / 4$.

Theorem 5.3. Each spacetime KSSdS[T] contains a unique (maximally extended) slicing $\mathcal{S}_{\tau}, \tau \in\left(\tau_{-}, \tau_{+}\right)$, such that

a. $\mathcal{S}_{\tau}$ is a compact CMC-hypersurface for all $\tau \in\left(\tau_{-}, \tau_{+}\right)$,

b. $\mathcal{S}_{\tau}$ is reflection symmetric for all $\tau$.

Along the slicing,

c. $K(\tau)$ is strictly monotonically increasing.

Every slicing $\mathcal{S}_{\tau}^{\prime}$ that satisfies (回) arises from $\mathcal{S}_{\tau}$ by combining the flow of $\mathcal{S}_{\tau}$ with an appropriate admixture of the Killing flow.

Remark. By definition, $\mathcal{T}$ is constant along the maximal integral curves of the oriented direction field on $\mathcal{K C}_{0}$. Below we prove that, for each $\mathrm{T}$, the equation $\mathcal{T}=\mathrm{T}$ defines a unique integral curve, which corresponds to a unique slicing $\mathcal{S}_{\tau}$ in $\operatorname{KSSdS}[\mathrm{T}], \tau \in\left(\tau_{-}, \tau_{+}\right)$, satisfying (国) $-(\mathbb{C})$. The function $\mathcal{T}$ can thus be viewed as a "Hamilton function" for the oriented direction field on $\mathcal{K C}_{0}$.

Proof. Each maximal integral curve of the oriented direction field on $\mathcal{K C}_{0}$ has a unique point of intersection with the line $K=$ const, since $\dot{K}>0$ everywhere. Hence the pairs $(0, C)$ with $C \in\left(C_{b}(0), C_{t}(0)\right)$ parametrize the family of integral curves in $\mathcal{K}_{0}$. In the following we prove that $\partial \mathcal{T}(K, C) / \partial C$ is positive for all $(K, C) \in \mathcal{K}_{0}$. By virtue of the asymptotic properties of $\mathcal{T}(K, C)$ established in Lemma 5.1 this implies that $C \mapsto \mathcal{T}(0, C)$ is a bijection between $\left(C_{b}(0), C_{t}(0)\right)$ and $\mathbb{R}$. Hence, there exists a unique pair $(0, C) \in \mathcal{K} \mathrm{C}_{0}$, such that $\mathcal{T}(0, C)=\mathrm{T}$, 
and thus a unique maximal integral curve in $\mathcal{K C}_{0}$, such that $\mathcal{T}=\mathrm{T}$ along the curve. Since the integral curve uniquely corresponds to a slicing $\mathcal{S}_{\tau}$ satisfying (国)-(価), the claim of the theorem is established. We now show that $\partial \mathcal{T}(K, C) / \partial C>0$ for all $(K, C) \in \mathcal{K C}_{0}$.

Consider the initial data generated by $\left(K_{\mathcal{S}}, C_{\mathcal{S}}\right) \in \mathcal{K C}_{0}$ and let again $L_{\mathcal{S}}=L\left(K_{\mathcal{S}}, C_{\mathcal{S}}\right)$, $\mathrm{T}=$ $\mathcal{T}\left(K_{\mathcal{S}}, C_{\mathcal{S}}\right)$. The universal covering of the data is embedded as a Cauchy CMC-hypersurface $\mathcal{S}$ in the covering space KSSdS. In a neighborhood of $\mathcal{S}$ we introduce Gaussian coordinates $(\sigma, \lambda)$, cf. the proof of Theorem 4.1] Now consider the (auxiliary) spacetime given by $(-\bar{\sigma}, \bar{\sigma}) \times$ $\left(-L_{\mathcal{S}}-\varepsilon, L_{\mathcal{S}}+\varepsilon\right) \times S^{2}$, which we denote by KSSdS[T]'; it is an open subset of KSSdS and spatially incomplete. In KSSdS $[\mathrm{T}]^{\prime}$ the hypersurface $\mathcal{S}$ does not generate a unique slicing, but evolves into a one-parameter family of slicings; we are able to show this in a straightforward way by using the methods of the proof of Theorem 4.1. We define the mean curvature operator $\mathcal{K}$ as in (30) and an operator $\mathcal{C}$,

$$
\mathcal{C}^{2}[\varphi]:=\frac{r^{6}(\varphi(\lambda), \lambda)}{6}\left(\mathcal{K}_{i j}[\varphi] \mathcal{K}^{i j}[\varphi]-\frac{\mathcal{K}[\varphi]^{2}}{3}\right)
$$

which assigns to every hypersurface $\sigma=\varphi(\lambda)$ a function $\left(-L_{\mathcal{S}}-\varepsilon, L_{\mathcal{S}}+\varepsilon\right) \ni \lambda \mapsto C[\varphi](\lambda)$; for a CMC-hypersurface characterized by $(K, C), \mathcal{C}[\varphi] \equiv C$. By construction, $\mathcal{K}[0]=K_{\mathcal{S}}$ and $\mathcal{C}[0]=C_{\mathcal{S}} . \mathcal{K}$ and $\mathcal{C}$ are $\mathcal{C}^{1}$ operators on $H_{\text {even }}^{2}\left(\left(-L_{\mathcal{S}}-\varepsilon, L_{\mathcal{S}}+\varepsilon\right)\right), \mathcal{K}^{\prime}[0]=\Delta+a$, cf. (34), and

$$
\mathcal{C}^{\prime}[0]=\frac{r^{3}}{3} \Delta-\nabla^{i}\left(\frac{r^{3}}{3}\right) \nabla_{i}+b
$$

where $b(\lambda)=M+K C / 3-4 C^{2} / r^{3}$ and $\nabla^{i}\left(r^{3} / 3\right) \nabla_{i}=r^{2} r^{\prime} \partial_{l}$. The set ker $\mathcal{K}^{\prime}[0]$ is not trivial, and neither is $\operatorname{ker} \mathcal{C}^{\prime}[0]$. However, the joint map $\left(\mathcal{K}^{\prime}, \mathcal{C}^{\prime}\right)[0]$ is an isomorphism; see Appendix $\mathbb{B}$ We may apply the inverse function theorem: there exists a unique continuously differentiable mapping $(\mathcal{K}, \mathcal{C})^{-1}$ such that $(\mathcal{K}, \mathcal{C})\left[(\mathcal{K}, \mathcal{C})^{-1}((\kappa, \gamma))\right]=(\kappa, \gamma)$ for all $(\kappa, \gamma)$ of a neighborhood of $\left(K_{\mathcal{S}}, C_{\mathcal{S}}\right)$ in $H_{\text {even }}^{0} \times H_{\text {even }}^{0}$. Hence, given a smooth function $(-\bar{\tau}, \bar{\tau}) \ni \tau \mapsto(K(\tau), C(\tau))$ such that $(K(0), C(0))=\left(K_{\mathcal{S}}, C_{\mathcal{S}}\right)$, a unique slicing $\mathcal{S}_{\tau}$ in $\mathrm{KSSdS}[\mathrm{T}]^{\prime}$ is defined by using $\varphi_{\tau}:=$ $(\mathcal{K}, \mathcal{C})^{-1}(K(\tau), C(\tau))$. By construction, the slicing $\mathcal{S}_{\tau}$ in $\mathrm{KSSdS}[\mathrm{T}]^{\prime}$ uniquely corresponds to a curve $(K, C)(\tau)$ in a neighborhood of $\left(K_{\mathcal{S}}, C_{\mathcal{S}}\right) \in \mathcal{K C}_{0}$.

Consider the hypersurface $\mathcal{S}$ and the slicing $\mathcal{S}_{\tau}$ in KSSdS[T] $]^{\prime}$ such that $\mathcal{S}_{0}=\mathcal{S}$ and $K(\tau)=K_{\mathcal{S}}$ for all $\tau$; the slicing is represented by a curve $(K, C)(\tau)=\left(K_{\mathcal{S}}, C(\tau)\right), C(0)=C_{\mathcal{S}}$, in $\mathcal{K C}_{0}$. Let $\alpha(\tau, l)$ be the lapse function of the slicing and $x(\tau, l)$ the shift vector in an arbitrary gauge. Making use of (44) we find that

$$
\frac{\partial r^{\prime}}{\partial \tau}\left(0, L_{\mathcal{S}}\right)=\frac{\partial^{2} r}{\partial l \partial \tau}\left(0, L_{\mathcal{S}}\right)=\left(\frac{K_{\mathcal{S}} r_{\max }}{3}-\frac{C_{\mathcal{S}}}{r_{\max }^{2}}\right) \alpha^{\prime}\left(0, L_{\mathcal{S}}\right)+x\left(0, L_{\mathcal{S}}\right) r_{\max }^{\prime \prime}
$$

where $r_{\max }=r\left(0, L_{\mathcal{S}}\right)$ and $r_{\max }^{\prime \prime}=r^{\prime \prime}\left(0, L_{\mathcal{S}}\right)$. Differentiation of the equation $r^{\prime}(\tau, L(\tau))=0$ results in

$$
\dot{L}(0)=-\left(\frac{K_{\mathcal{S}} r_{\max }}{3}-\frac{C_{\mathcal{S}}}{r_{\max }^{2}}\right) \frac{\alpha^{\prime}\left(0, L_{\mathcal{S}}\right)}{r_{\max }^{\prime \prime}}-x\left(0, L_{\mathcal{S}}\right) .
$$

We are now prepared to investigate the derivative of $\mathcal{T}(\tau)$ at $\tau=0$. The definition $\mathcal{T}(\tau)=$ $t(\tau, L(\tau))$ leads to

$$
\dot{\mathcal{T}}(0)=\frac{\partial t}{\partial \tau}\left(0, L_{\mathcal{S}}\right)+\frac{\partial t}{\partial l}\left(0, L_{\mathcal{S}}\right) \dot{L}(0)=\frac{\alpha^{\prime}\left(0, L_{\mathcal{S}}\right)}{r_{\max }^{\prime \prime}}
$$


where we have employed (24) and (44). By virtue of Eq. (53) (which holds also for slicings of $\operatorname{KSSdS}[\mathrm{T}]^{\prime}$ ), we have $\dot{C}(0)=r_{\min }^{\prime \prime} r_{\min }^{2} \alpha(0,0)$, and thus

$$
\left.\frac{\partial \mathcal{T}(K, C)}{\partial C}\right|_{\left(K_{\mathcal{S}}, C_{\mathcal{S}}\right)}=\frac{1}{r_{\min }^{2} r_{\min }^{\prime \prime 2}} \frac{r_{\min }^{\prime \prime}}{r_{\max }^{\prime \prime}} \frac{\alpha^{\prime}\left(0, L_{\mathcal{S}}\right)}{\alpha(0,0)}=\frac{r_{\min }^{\prime \prime}}{r_{\max }^{\prime \prime}} \frac{\alpha_{0}^{\prime}\left(L_{\mathcal{S}}\right)}{r_{\min }^{2} r_{\min }^{\prime \prime 2}}=\frac{1}{2} \frac{\alpha_{0}^{\prime}\left(2 L_{\mathcal{S}}\right)}{r_{\min }^{2} r_{\min }^{\prime \prime 2}},
$$

where we have also used (100) from App. B $\alpha_{0}$ denotes the solution of the homogeneous lapse equation (37), associated with $\left(K_{\mathcal{S}}, C_{\mathcal{S}}\right)$, with initial data $\left.\alpha_{0}\right|_{l=0}=1$. By virtue of Lemma 4.2. $\alpha_{0}$ is not periodic, so that $\alpha_{0}^{\prime}\left(2 L_{\mathcal{S}}\right) \neq 0$; moreover, since $\alpha_{0}^{\prime}\left(2 L_{\mathcal{S}}\right) \neq 0$ holds irrespective of the choice of $(K, C)=\left(K_{\mathcal{S}}, C_{\mathcal{S}}\right)$, it has a definite sign for all $(K, C) \in \mathcal{K C}_{0}$. Consequently, also $\partial \mathcal{T}(K, C) / \partial C \gtrless 0$ for all $(K, C)$. Indeed $\partial \mathcal{T} / \partial C>0$ by virtue of the asymptotic behavior of $\mathcal{T}(K, C)$ described in Lemma [5.1. This establishes the claim of the theorem.

Remark. The slicing $\mathcal{S}_{\tau}$ in KSSdS[0], which constitutes the only time-symmetric spacetime in the family KSSdS[T], is represented by a distinguished integral curve in $\mathcal{K C}_{0}$ that is invariant under the inversion. The curve passes through the origin $(0,0)$ which is associated with a CMC-hypersurface of time-symmetry, the hypersurface $t=0$, which connects the bifurcation 2-spheres.

Remark. The approach we have taken to establish the main results displays a pronounced interplay between the geometric analysis and the analysis of the function $\mathcal{T}$. We have chosen this approach because the direct investigation of this function is non-trivial; e.g. it is not straightforward to show directly that it has no critical points, since methods like the ones applied in [3] fail.

Theorem 5.4. In KSSdS[T] consider the unique maximally extended slicing $\mathcal{S}_{\tau}, \tau \in\left(\tau_{-}, \tau_{+}\right)$, satisfying the properties (ㅁ)-(ㄷ) of Theorem 5.3. There exists an interval $\left(\bar{\tau}_{-}, \bar{\tau}_{+}\right) \subseteq\left(\tau_{-}, \tau_{+}\right)$, such that $\mathcal{S}_{\tau}, \tau \in\left(\bar{\tau}_{-}, \bar{\tau}_{+}\right)$, is a foliation.

Proof. Consider the lapse equation (38) for an umbilical pair $(K, C) \in \mathcal{K C}_{0}$, i.e. $C=0$. In this case $a=\Lambda-K^{2} / 3=$ const, and

$$
\left.\alpha\right|_{(K, C=0)}=\dot{K}\left(\Lambda-\frac{K^{2}}{3}\right)^{-1}=\text { const }>0
$$

is the unique even solution on the domain $S^{1}$ provided by Corollary 4.3 Let $\alpha(\tau, l)$ be the lapse function of the slicing $\mathcal{S}_{\tau}$ in $\mathrm{KSSdS}[\mathrm{T}]$ which is represented by $(K, C)(\tau)$ in $\mathcal{K C}_{0}$. The asymptotic properties (61) imply that there exists $\tau_{0}$ such that $C\left(\tau_{0}\right)=0$. At $\tau=\tau_{0}$, $\alpha$ is given by (70), i.e. $\alpha\left(\tau_{0}, l\right)=$ const $>0$. Since $\alpha(\tau, l)$ continuously depends on $\tau$, cf. (41), there exists an interval $\left(\bar{\tau}_{-}, \bar{\tau}_{+}\right) \ni \tau_{0}$, such that $\alpha\left(\tau_{0}, l\right)>0$ for all $\tau \in\left(\bar{\tau}_{-}, \bar{\tau}_{+}\right)$. Hence $\mathcal{S}_{\tau}$ is a foliation for $\tau \in\left(\bar{\tau}_{-}, \bar{\tau}_{+}\right)$.

Remark. Since the lapse function is explicitly known for $C=0$, the direction field $(\dot{K}, \dot{C})$ at $C=0$ can be computed explicitly as well. From (170) we obtain

$$
\left.\dot{C}\right|_{(K, C=0)}=\dot{K} M\left(\Lambda-\frac{K^{2}}{3}\right)^{-1}=\text { const }>0 \text {. }
$$

We infer that every integral curve intersects the line $C=0$ exactly once, hence the value of $\tau_{0}$ introduced in the proof of Theorem 5.4 is unique. 
In general, $\left(\bar{\tau}_{-}, \bar{\tau}_{+}\right) \neq\left(\tau_{-}, \tau_{+}\right)$in Theorem [5.4 this is proved in Appendix [C] In particular, Corollary C.3 shows that $\left(\bar{\tau}_{-}, \bar{\tau}_{+}\right) \neq\left(\tau_{-}, \tau_{+}\right)$for all spacetimes KSSdS[T] with $|\mathrm{T}|$ large enough.

Conjecture 5.5. There exist numbers $0<F_{c}<F_{0}<1$ such that the following statement holds: if KSSdS[T] is a cosmological Kottler-Schwarzschild-de Sitter spacetime with $9 M^{2} \Lambda \in$ $\left[F_{c}, F_{0}\right)$ and sufficiently small $|T|$, then the maximally extended slicing $\mathcal{S}_{\tau}, \tau \in\left(\tau_{-}, \tau_{+}\right)$, of Theorem (5.3) is a foliation.

This conjecture is based on extensive numerical evidence, which we present in Appendix C

\section{Acknowledgements}

We would like to thank Christina Stanciulescu whose work [10] and handwritten notes were a very useful starting point for the present paper. J.M.H. would also like to thank Alan Rendall for helpful discussions.

\section{A The parameter space $\mathcal{K C}_{0}$ of compact CMC-data}

\section{A.1 Properties of $\mathcal{K}_{0}$}

By definition, $(K, C) \in \mathcal{K C}$ iff there exists a neighborhood of $(K, C) \in \mathbb{R}^{2}$ such that all pairs of that neighborhood generate compact CMC-initial data. The function $r(l)$ that determines the initial data is given by the equation

$$
r^{\prime}= \pm \sqrt{D(r)} \quad \text { with } \quad D(r)=D(K, C ; r)=1-\frac{2 \bar{M}}{r}-\frac{\bar{\Lambda} r^{2}}{3}+\frac{C^{2}}{r^{4}},
$$

where $\bar{M}=M+C K / 3$ and $\bar{\Lambda}=\Lambda-K^{2} / 3$; the initial data is compact iff $r(l)$ is periodic and thus interpretable as a function $S^{1} \rightarrow \mathbb{R}$. Therefore, $(K, C) \in \mathcal{K} \mathcal{C}$ iff the function $D(r)$ possesses two positive (simple) zeros $r_{\min }$ and $r_{\max }$, such that $D(r)>0$ in $\left(r_{\min }, r_{\max }\right)$. (Accordingly, when viewed over $r \in\left[r_{\min }, r_{\max }\right], r^{\prime}= \pm \sqrt{D(r)}$ describes a closed curve, cf. Fig. 3(b), and $r(l)$ becomes a periodic function which oscillates between $r_{\min }$ and $r_{\max }$.)

Assume $C \neq 0$; then $D(r) \rightarrow \infty$ as $r \rightarrow 0$, thus two positive extrema are necessary to obtain the desired profile of $D(r)$, cf. Fig. 3(a). The critical points of $D(r)$ are

$$
\left(r_{-}\right)^{3}=\frac{3}{2 \bar{\Lambda}}\left(\bar{M}-\sqrt{\bar{M}^{2}-\frac{8}{3} \bar{\Lambda} C^{2}}\right) \quad\left(r_{+}\right)^{3}=\frac{3}{2 \bar{\Lambda}}\left(\bar{M}+\sqrt{\bar{M}^{2}-\frac{8}{3} \bar{\Lambda} C^{2}}\right),
$$

which are positive, iff

$$
\bar{\Lambda}>0 \quad, \quad \bar{M}>0 \quad, \quad C^{2}<\frac{3}{8} \frac{\bar{M}^{2}}{\bar{\Lambda}} .
$$

For future reference we note that

$$
\bar{\Lambda}>0 \quad \Leftrightarrow \quad K \in(-\sqrt{3 \Lambda},+\sqrt{3 \Lambda}) .
$$


When the conditions (74) are satisfied, $r_{-} / r_{+}$is automatically a minimum/maximum of $D(r)$; however, the conditions are not sufficient to ensure the profile $3(\mathrm{a})$ of $D(r)$.

Note that the case $C=0$ is simpler: $D(r)$ possesses the desired profile iff $\bar{\Lambda}>0$. Proof: $D(r) \rightarrow-\infty(r \rightarrow 0, r \rightarrow \infty) ; r_{-}$does not exist; $r_{+}=\sqrt[3]{3 M / \bar{\Lambda}}$ is a maximum iff $\bar{\Lambda}>0$. Moreover, $D\left(r_{+}\right)>0$, since $9 M^{2} \bar{\Lambda} \leq 9 M^{2} \Lambda<1$.

We can effectively reduce the problem by one parameter by writing

$$
\tilde{D}(\tilde{r})=1-\frac{2 \tilde{M}}{\tilde{r}}-\frac{\tilde{r}^{2}}{3}+\frac{\tilde{C}^{2}}{\tilde{r}^{4}} \quad \text { with } \quad \tilde{r}=\sqrt{\bar{\Lambda}} r, \quad \tilde{M}=\bar{M} \sqrt{\bar{\Lambda}}, \quad \tilde{C}=C \bar{\Lambda} .
$$

Investigating (76) we find that the function $\tilde{D}(\tilde{r})$ has the desired profile if and only if $(\tilde{M}, \tilde{C})$ lies in a certain connected open set whose boundaries are convex/concave functions. Using these properties, via the variable transformation relating $(\tilde{M}, \tilde{C})$ and $(K, C)$ for given $(M, \Lambda)$ we can prove

$$
\mathcal{K C}=\mathcal{K C}_{0} \cup \mathcal{K} \mathfrak{C}_{1} \cup \mathcal{K} \mathcal{C}_{-1},
$$

where the $\mathcal{K C}_{i}$ are pairwise disjoint connected open sets; $\mathcal{K} \mathcal{C}_{-1}$ arises from $\mathcal{K C}_{1}$ by inversion at the origin. $\mathcal{K C}_{0}$ is the connection component of $(K, C)=(0,0)$, it is invariant under the inversion, cf. Fig. 4. $\mathcal{K C}_{0}$ is enclosed by the functions $C_{t}(K)$ and $C_{b}(K)=-C_{t}(-K)$, and the vertical straight lines $K= \pm \sqrt{3 \Lambda}$.

The functions $C_{b, t}(K)$ are only know implicitly; the defining equation for $C_{b, t}$ is $D\left(r_{-}\right)=0$, or, equivalently, $\bar{\Lambda} r_{-}^{3}+3 \bar{M}=2 r_{-}$, where $\bar{\Lambda}, \bar{M}$, and $r_{-}$depend on $\left(K, C_{t, b}(K)\right)$. In the limit of small $\bar{\Lambda}$ we obtain the approximate solutions

$$
C_{b, t}(K)=\frac{\sqrt{3}}{K^{2}}( \pm 1 \mp \sqrt{1 \mp \sqrt{3} K M})^{2}\left( \pm 1 \pm \frac{3}{2} \frac{\bar{\Lambda}}{K^{2}} \frac{( \pm 1 \mp \sqrt{1 \mp \sqrt{3} K M})^{2}}{\sqrt{1 \mp \sqrt{3} K M}}\right)+O\left(\bar{\Lambda}^{2}\right),
$$

where the upper sign applies to $C_{t}$, the lower to $C_{b}$; note that $\left|C_{b}(\sqrt{3 \Lambda})\right|<\left|C_{t}(\sqrt{3 \Lambda})\right|$.

The functions $C_{b, t}$ are strictly monotonically increasing. To show this we differentiate the defining equation $D\left(r_{-}\right)=0$; we obtain

$$
\frac{\partial C_{b, t}}{\partial K}=\left.\frac{1}{3} r_{-}^{3}\right|_{C_{b, t}} .
$$

For the sake of completeness we note that $C_{t}(K)$ is a convex function.

\section{A.2 $\quad r_{\min }$ and $r_{\max }$ on $\mathcal{K C}_{0}$}

We investigate $r_{\min }$ and $r_{\max }$ as functions of $(K, C) \in \overline{\mathcal{K} \bigodot_{0}}$. In the special case $C=0$, the function $D(r)$ reduces to $D(r)=1-2 M / r-\bar{\Lambda} r^{2} / 3$, thus

$$
r_{\min }=\frac{2}{\sqrt{\bar{\Lambda}}} \cos \left(\frac{\xi+\pi}{3}\right), \quad r_{\max }=\frac{2}{\sqrt{\bar{\Lambda}}} \cos \left(\frac{\xi-\pi}{3}\right) \quad \text { where } \quad \cos \xi:=3 M \sqrt{\bar{\Lambda}} .
$$

Two subcases deserve special attention: when $K=0$ we have $\cos \xi=3 M \sqrt{\Lambda}$ and $r_{\min }, r_{\max }$ coincide with the horizons of Schwarzschild-de Sitter, $r_{\min }=r_{\mathrm{b}}, r_{\max }=r_{\mathrm{c}}$. In the limit 
$K \rightarrow \pm \sqrt{3 \Lambda},(79)$ becomes

$$
r_{\min }=2 M+\frac{8}{3} M^{3} \bar{\Lambda}+O\left(\bar{\Lambda}^{2}\right) \quad, \quad r_{\max }=\frac{\sqrt{3}}{\sqrt{\bar{\Lambda}}}+O(\sqrt{\bar{\Lambda}}) .
$$

Eq. (179) shows that $r_{\min } \downarrow$ and $r_{\max } \uparrow$ when $|K| \uparrow$; hence, for $C=0$, for all $K, r_{\min } \leq r_{\mathrm{b}}$ and $r_{\max } \geq r_{\mathrm{c}}$.

By recalling that $D(r)=V(r)+\left[K r / 3-C / r^{2}\right]^{2}$, we conclude from $V(r)>0 \forall r \in\left(r_{\mathrm{b}}, r_{\mathrm{c}}\right)$ that $r_{\min }, r_{\max } \in\left(r_{\mathrm{b}}, r_{\mathrm{c}}\right)$ is excluded. Since $r_{\min } \leq r_{\mathrm{b}}$ and $r_{\max } \geq r_{\mathrm{c}}$ for $C=0$, and since $r_{\min }$ and $r_{\max }$ are continuous functions of $(K, C)$ on the connected domain $\overline{\mathcal{K} \mathcal{C}_{0}}, r_{\text {min }} \leq r_{\mathrm{b}}$ and $r_{\max } \geq r_{\mathrm{c}}$ must hold everywhere on $\overline{\mathcal{K} \mathcal{C}_{0}}$.

Suppose that $r_{\min }=r_{\mathrm{b}}$ for some $(K, C) \in \overline{\mathcal{K} \mathcal{C}_{0}}$; it then follows from $D\left(r_{\mathrm{b}}\right)=0$ that $C=$ $\left(r_{\mathrm{b}}^{3} / 3\right) K$. Conversely, consider $(K, C)$ with $C=\left(r_{\mathrm{b}}^{3} / 3\right) K$; from

$$
D(r)=1-\frac{2 M}{r}-\frac{\Lambda r^{2}}{3}+\left(\frac{K r}{3}-\frac{K r_{\mathrm{b}}^{3}}{3 r^{2}}\right)^{2}
$$

we obtain $D\left(r_{\mathrm{b}}\right)=0$ and $d D /\left.d r\right|_{r_{\mathrm{b}}}>0$, therefore $r_{\min }=r_{\mathrm{b}}$. Hence

$$
r_{\min }(K, C)=r_{\mathrm{b}} \quad \Longleftrightarrow \quad C=\frac{r_{\mathrm{b}}^{3}}{3} K .
$$

We conclude that there exists a straight line in $\overline{\mathcal{K} \mathcal{C}_{0}}$, given by $C=\left(r_{\mathrm{b}}^{3} / 3\right) K$, along which $r_{\text {min }}$ attains the maximal possible value $r_{\mathrm{b}}$. This straight line intersects the boundary $\partial\left(\mathcal{K C}_{0}\right)$ in the $K= \pm \sqrt{3 \Lambda}$ vertical lines (and has no intersection with $C_{b, t}$ ). To establish this result we verify that $\left(r_{\mathrm{b}}^{3} / 3\right) \sqrt{3 \Lambda}<C_{t}(\sqrt{3 \Lambda})$ and we note that $r_{-}<r_{\mathrm{b}}$ everywhere, so that the slope of $C_{t}(K)$, cf. (78), is always less than the slope of the straight line. Hence, this "line of maximal $r_{\text {min }}$ " divides $\overline{\mathcal{K C}_{0}}$ into two regions, an upper (left) half and a lower (right) half. In each of the two halves $\left[K r_{\min } / 3-C / r_{\min }^{2}\right] \neq 0$ holds, since $\left[K r_{\min } / 3-C / r_{\min }^{2}\right]= \pm \sqrt{-V\left(r_{\min }\right)}$; it follows from the connectedness of the regions and the continuity of the function that $\left[K r_{\min } / 3-C / r_{\min }^{2}\right]<0$ in the upper left half and $\left[K r_{\min } / 3-C / r_{\min }^{2}\right]>0$ in the lower right half, see Fig. 10(a).

Analogously, we find a straight line of minimal $r_{\text {max }}$ in $\overline{\mathcal{K} \mathcal{C}_{0}}$,

$$
r_{\max }(K, C)=r_{\mathrm{c}} \quad \Longleftrightarrow \quad C=\frac{r_{\mathrm{c}}^{3}}{3} K .
$$

The straight line of minimal $r_{\max }, C=\left(r_{\mathrm{c}}^{3} / 3\right) K$, intersects $C_{b, t}(K)$ and so defines an (upper) left half of $\overline{\mathcal{K} C_{0}}$, where $\left[K r_{\max } / 3-C / r_{\text {max }}^{2}\right]<0$ and a (lower) right half, where $\left[K r_{\max } / 3-\right.$ $\left.C / r_{\text {max }}^{2}\right]>0$ holds, see Fig. 10(b).

Consider a curve $(K, C)(\nu)$ and regard $r_{\min }$ and $r_{\max }$ as functions of $\nu$. By differentiating the equation $D\left(r_{\min }\right)=0$ w.r.t. $\nu$ we obtain

$$
\dot{r}_{\min }=-2\left(\left.\frac{d D}{d r}\right|_{r_{\min }}\right)^{-1}\left[\frac{K r_{\min }}{3}-\frac{C}{r_{\min }^{2}}\right]\left[\frac{\dot{K} r_{\min }}{3}-\frac{\dot{C}}{r_{\min }^{2}}\right],
$$

and the analogous result for $r_{\max }$; the overdot denotes differentiation w.r.t. $\nu$. 

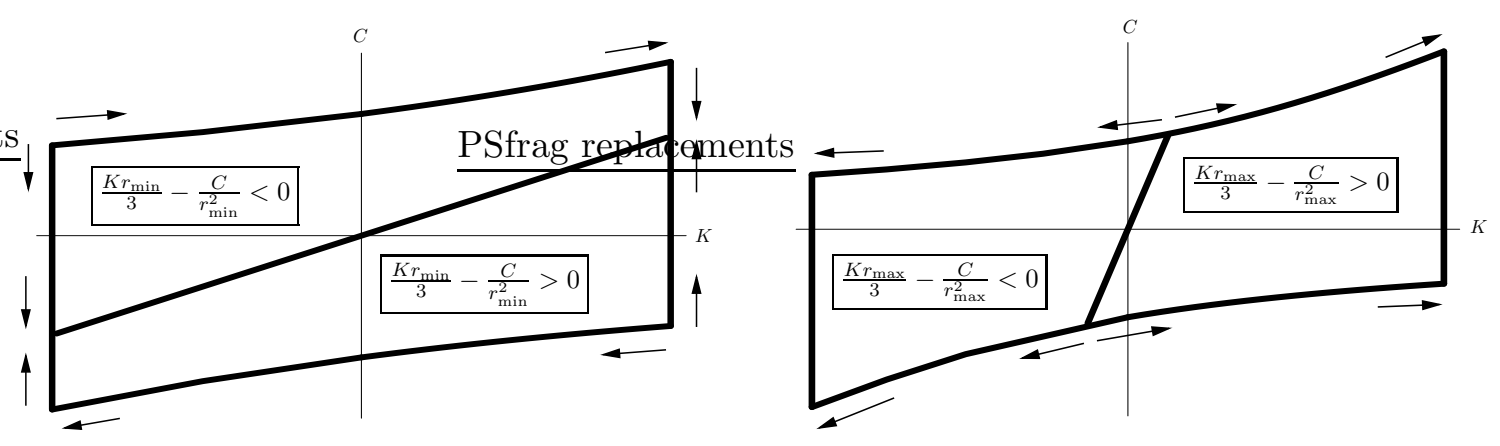

Figure 10: $r_{\text {min }}$ is maximal, $r_{\min }=r_{\mathrm{b}}$, along a straight line. It divides $\overline{\mathcal{K C}_{0}}$ in two halves, which are characterized by $K r_{\min } / 3-C / r_{\min }^{2} \gtrless 0$. On the boundaries, $r_{\min }$ increases as indicated by the arrows. For the first subfigure $\Lambda=1, M=1 / 5 . r_{\max }$ is minimal, $r_{\min }=r_{\mathrm{c}}$, along another straight line, which defines the regions $K r_{\max } / 3-C / r_{\max }^{2} \gtrless 0$. On the boundaries, $r_{\max }$ increases as indicated by the arrows; $r_{\max } \rightarrow \infty(K \rightarrow \pm \sqrt{3 \Lambda})$. For the second subfigure $\Lambda=1, M=3 / 10$.

Consider the boundaries $C_{b, t}$ as parametrized curves $\left(K(\nu), C_{b, t}(K(\nu))\right)$ and assume $\dot{K}>0$. By definition, $r_{\min }=r_{-}$on $C_{b, t}$, whereby $d D / d r=0$ at $r_{\min }$, so that (84) is not applicable. However, by differentiating the equation $d D /\left.d r\right|_{r_{\min }}=0$ we are able to express $\dot{r}_{\min }$ in terms of regular expressions: using $\dot{C}=\left(r_{\min }^{3} / 3\right) \dot{K}$, see (178), we obtain

$$
\dot{r}_{\min }=-2\left(\left.\frac{d^{2} D}{d r^{2}}\right|_{r_{\min }}\right)^{-1} \dot{K}\left[\frac{K r_{\min }}{3}-\frac{C}{r_{\min }^{2}}\right] \quad \text { along } C_{b, t} .
$$

Since $\left[K r_{\min } / 3-C / r_{\min }^{2}\right]<0$ on $C_{t}$ and $d^{2} D /\left.d r^{2}\right|_{r_{\min }}>0$ we observe that $\dot{r}_{\text {min }}>0$ along $C_{t}$; by virtue of the reflection symmetry, $\dot{r}_{\min }<0$ along $C_{b}$.

From the monotonicity properties of $r_{\min }$ along $C_{b, t}$ and the lines $K= \pm \sqrt{3 \Lambda}$, we conclude that $r_{\text {min }}$ assumes its global minimum at the point $(K, C)=\left(\sqrt{3 \Lambda}, C_{b}(\sqrt{3 \Lambda})\right)$ and at the reflected point, see Fig. 10(a)

Using (78) in the $r_{\text {max-analogue of (84) }}$ we get

$$
\dot{r}_{\max }=-2\left(\left.\frac{d D}{d r}\right|_{r_{\max }}\right)^{-1}\left[\frac{K r_{\max }}{3}-\frac{C}{r_{\max }^{2}}\right] \frac{\dot{K} r_{\max }}{3}\left[1-\frac{r_{-}^{3}}{r_{\max }^{3}}\right] \quad \text { along } C_{b, t} .
$$

We infer the properties of $r_{\max }$ along $C_{b, t}$ as depicted in Fig. 10(b) In contrast to $r_{\min }, r_{\max }$ is unbounded as $K \rightarrow \sqrt{3 \Lambda}$.

For small $\bar{\Lambda}$ we can calculate $r_{\min }$ and $r_{\max }$ along $C_{b, t}$ up to any desired order of $\bar{\Lambda}$ :

$$
r_{\text {min }}= \pm \frac{\sqrt{3}}{K}( \pm 1 \mp \sqrt{1 \mp \sqrt{3} K M})\left( \pm 1+\frac{3 \sqrt{3} M}{4} \frac{\bar{\Lambda}}{K} \frac{( \pm 1 \mp \sqrt{1 \mp \sqrt{3} K M})}{\sqrt{1 \mp \sqrt{3} K M}}\right)+O\left(\bar{\Lambda}^{2}\right)
$$

along $C_{b, t}$ for $\bar{\Lambda} \rightarrow 0$, where the upper sign applies to $C_{t}$, the lower sign to $C_{b}$. Setting $\bar{\Lambda}=0$, Eq. (87) becomes

$$
r_{\min }=\frac{1}{\sqrt{\Lambda}}( \pm 1 \mp \sqrt{1 \mp 3 \sqrt{\Lambda} M}) \quad \text { for } K=\sqrt{3 \Lambda}, C=C_{b, t}(\sqrt{3 \Lambda}) .
$$


Finally, along $C_{b, t}, r_{\max }$ is given by

$$
r_{\max }=\frac{2}{\sqrt{\bar{\Lambda}}} \cos \left(\frac{\bar{\xi}-\pi}{3}\right)+O\left(\bar{\Lambda}^{3 / 2}\right) \quad \text { where } \quad \cos \bar{\xi}:=3 \bar{M} \sqrt{\bar{\Lambda}},
$$

which follows when $C^{2} / r^{4}$ is neglected against $\bar{M} / r$ in $D(r)$.
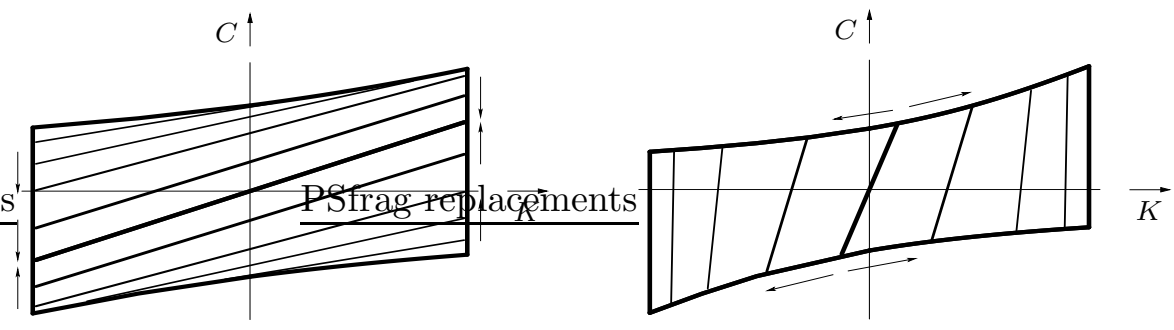

Figure 11: $r_{\min }$ and $r_{\max }$ are constant along straight lines in $\mathcal{K C}$. We chose $\Lambda=1$ and $M=1 / 5$, $M=3 / 10$.

For a given $r_{0} \in\left(\sqrt{\Lambda}^{-1}(1-\sqrt{1-3 \sqrt{\Lambda} M}), r_{\mathrm{b}}\right)$, the set of all $(K, C)$ such that $r_{\min }(K, C)=r_{0}$ is (a segment of) a straight line in $\overline{\mathcal{K} \mathcal{C}_{0}}: D\left(r_{0}\right)=0$ holds iff $(K, C)$ is chosen according to

$$
C=\frac{r_{0}^{3}}{3} K \mp r_{0}^{2} \sqrt{-V\left(r_{0}\right)}
$$

moreover, $d D /\left.d r\right|_{r_{0}}>0$ holds for (a segment of) the described straight line; hence $r_{\min }=r_{0}$ there. The lines of constant $r_{\min }$ are depicted in Fig. 11(a) Analogously, $r_{\max }$ is constant along straight lines (90), where $r_{0} \in\left(r_{\mathrm{c}}, \infty\right)$; see Fig. 11(b)]

\section{A.3 Hypersurfaces $r=$ const}

An alternative way of investigating the space $\mathcal{K C}$ is based on an analysis of the CMC-data associated with $r=$ const hypersurfaces. In KSSdS the hypersurfaces $r=$ const are spacelike hypersurfaces with unit normal $\sqrt{-V(r)} \partial / \partial r$ when $r \in\left(0, r_{\mathrm{b}}\right) \cup\left(r_{\mathrm{c}}, \infty\right)$. A hypersurface $r=$ const possesses constant mean curvature, the induced CMC-data is represented by the constants $K, C$,

$$
K=\mp \frac{1}{r \sqrt{-V(r)}}\left(-2+\frac{3 M}{r}+\Lambda r^{2}\right), \quad C= \pm \frac{r^{2}}{\sqrt{3}} \sqrt{1-\left(\Lambda-\frac{K^{2}}{3}\right) r^{2}},
$$

The induced metric is $d l^{2}+r^{2} d \Omega^{2}$ with $l=t \sqrt{-V(r)} \in(-\infty, \infty)$; identifying $l=l_{0}$ with $l=l_{1}>l_{0}$ leads to compact CMC-data, however, no canonical choice of $l_{0}, l_{1}$ exists.

The $r=$ const initial data sets play the role of borderline cases: $r=$ const data arises when the function $D(r)$ possesses a double zero. We infer that the pair $(K, C)$ of (191) lies on the boundary of $\mathcal{K C}_{0}$ :

$$
(K, C) \in C_{b, t} \quad \text { when } \frac{1}{\sqrt{\Lambda}}(1-\sqrt{1-3 \sqrt{\Lambda} M})<r<\frac{1}{\sqrt{\Lambda}}(-1+\sqrt{1+3 \sqrt{\Lambda} M}) .
$$

Eqs. (91) thus constitute a parametric representation of the boundaries $C_{b, t}$ of $\mathcal{K C}_{0}$. 


\section{B The lapse equation}

We consider the equation

$$
\Delta \alpha+a \alpha=0, \quad \text { i.e. } \quad \alpha^{\prime \prime}+\frac{2}{r} r^{\prime} \alpha^{\prime}+a \alpha=0,
$$

where $a(l)=\Lambda-K^{2} / 3-6 C^{2} / r^{6}(l)$; note that there do not exist pairs $(K, C) \in \mathcal{K C}_{0}$ such that $a(l)$ is non-positive, which follows from algebraic computations based on the results of Appendix $\mathbb{A}$. We seek the general solution on the domain $\mathbb{R}$. Equation (93) is homogeneous with periodic, even and odd coefficient functions. The general solution of (93) is a superposition of two principal solutions with symmetry properties:

- Let $\alpha_{0}(l)$ denote the function that solves (93) with initial conditions $\alpha_{0}(0)=1$ and $\alpha_{0}^{\prime}(0)=0$; it is even.

- The function $\alpha_{\xi}(l)=r^{\prime}(l)$ is odd; it solves (93) with initial conditions $\alpha_{\xi}(0)=0$, $\alpha_{\xi}^{\prime}(0)=r_{\min }^{\prime \prime}$.

The function $\alpha_{\xi}$ is positive in $(0, L)$ but negative in $(-L, 0) ; \alpha_{\xi}(0)=\alpha_{\xi}(L)=0 ; \alpha_{\xi}$ is a periodic function. Geometrically speaking, $\alpha_{\xi}(l)=r^{\prime}(l)$ is the lapse function associated with the Killing vector $\xi$, cf. (11).

To investigate the general solution of (93) we use Floquet's theorem. Let us write (93) as a system of first order,

$$
\left(\begin{array}{c}
\alpha \\
\alpha^{\prime}
\end{array}\right)^{\prime}=\left(\begin{array}{cc}
0 & 1 \\
-a & -2 r^{\prime} / r
\end{array}\right)\left(\begin{array}{c}
\alpha \\
\alpha^{\prime}
\end{array}\right)=A(l)\left(\begin{array}{c}
\alpha \\
\alpha^{\prime}
\end{array}\right)
$$

The principal solution matrix is given by

$$
\Pi(l, 0)=\left(\begin{array}{cc}
\alpha_{0}(l) & r^{\prime}(l) / r_{\min }^{\prime \prime} \\
\alpha_{0}^{\prime}(l) & r^{\prime \prime}(l) / r_{\min }^{\prime \prime}
\end{array}\right), \quad \Pi^{\prime}(l, 0)=A(l) \Pi(l, 0), \quad \Pi(0,0)=\left(\begin{array}{ll}
1 & 0 \\
0 & 1
\end{array}\right) .
$$

The Wronskian $W(l, 0)=\operatorname{det} \Pi(l, 0)$ satisfies the equation $W^{\prime}(l, 0)=(\operatorname{tr} A(l)) W(l, 0)$, thus

$$
W(l, 0)=\operatorname{det} \Pi(l, 0)=\exp \int_{0}^{l} \operatorname{tr} A(\tilde{l}) d \tilde{l}=r_{\min }^{2} / r(l)^{2} .
$$

From $W(2 L, 0)=1$ we see that the so-called monodromy matrix $\Pi(2 L, 0)$ fulfills

$$
\Pi(2 L, 0)=\left(\begin{array}{cc}
1 & 0 \\
\alpha_{0}^{\prime}(2 L) & 1
\end{array}\right)
$$

Floquet's theorem states that $\Pi(l, 0)=P(l, 0) \exp (l Q(0))$, where $P(l, 0)$ is a periodic matrix and $Q(0)$ is such that $\exp (2 L Q(0))=\Pi(2 L, 0)$. Thus,

$$
\Pi(l, 0)=P(l, 0) \exp \left[l\left(\begin{array}{cc}
0 & 0 \\
\alpha_{0}^{\prime}(2 L) /(2 L) & 0
\end{array}\right)\right]=P(l, 0)\left(\begin{array}{cc}
1 & 0 \\
\left(l \alpha_{0}^{\prime}(2 L)\right) /(2 L) & 1
\end{array}\right) .
$$


It follows that

$$
\alpha_{0}(2 n L+l)=\alpha_{0}(l)+n\left[\alpha_{0}^{\prime}(2 L) / r_{\min }^{\prime \prime}\right] \alpha_{\xi}(l) \quad \forall l, \quad \forall n \in \mathbb{Z},
$$

in particular, $\alpha_{0}(2 n L)=\alpha_{0}(0)=1$. When we employ the symmetry properties of the coefficients in the differential equation (94) we are able to also establish a direct relation between the initial data at $l=0$ and the solution at the half-period $l=L$ :

$$
\Pi(L, 0)=\left(\begin{array}{cc}
\frac{r_{\min }^{\prime \prime}}{r_{\max }^{\prime \prime}} \frac{r_{\min }^{2}}{r_{\max }^{2}} & 0 \\
\alpha_{0}^{\prime}(L) & \frac{r_{\max }^{\prime \prime}}{r_{\min }^{\prime \prime}}
\end{array}\right), \quad \text { where } \quad \alpha_{0}^{\prime}(2 L)=2 \alpha_{0}^{\prime}(L) \frac{r_{\min }^{\prime \prime}}{r_{\max }^{\prime \prime}} .
$$

We infer that $\alpha_{0}(l)$ (and thus the general solution of (93)) is periodic iff $\alpha_{0}^{\prime}(L)=0$. We prove $\alpha_{0}^{\prime}(L) \neq 0$, which implies that the even solutions of (93) are not periodic, so that Lemma 4.2 is established.

We analyze an explicit representation of $\alpha_{0}$, which we obtain via an ansatz $\alpha_{0}(l)=\beta(l) r^{\prime}(l)$; we get

$$
\alpha_{0}(l)=-\dot{C} r^{\prime} \int^{l} \frac{1}{r^{2} r^{\prime 2}} d l+k r^{\prime},
$$

where $\dot{C}$ abbreviates $\dot{C}=r_{\min }^{2} r_{\min }^{\prime \prime}$, and $k=$ const is such that the function is even. The integral diverges as $l \rightarrow 0$ and $l \rightarrow \pm L$; however, by applying de l'Hospital's rule it is confirmed that $\alpha_{0}(0)=1$ and $\alpha_{0}( \pm L)= \pm\left(r_{\min }^{\prime \prime} / r_{\max }^{\prime \prime}\right)\left(r_{\min }^{2} / r_{\max }^{2}\right)$, cf. (100).

When we view (the first half-period of) the function $\alpha_{0}$ as a function of $r$ we can write

$$
\alpha_{0}(r)=\dot{C} \sqrt{D(r)}\left(-\int^{r} \frac{1}{\hat{r}^{2}} \frac{1}{D(\hat{r})^{3 / 2}} d \hat{r}+k\right) .
$$

We introduce $\delta(r)$ by defining $\delta(r)=r^{4} D(r)\left(r-r_{\min }\right)^{-1}\left(r_{\max }-r\right)^{-1}$; the function $\delta(r)$ is positive in $\left[r_{\min }, r_{\max }\right]$. Define

$$
\phi(r)=\frac{4 r-2\left(r_{\max }+r_{\min }\right)}{\left(r_{\max }-r_{\min }\right)^{2} \sqrt{\left(r-r_{\min }\right)\left(r_{\max }-r\right)}}=\int \frac{1}{\left(r-r_{\min }\right)^{3 / 2}\left(r_{\max }-r\right)^{3 / 2}} d r,
$$

then $\alpha_{0}(r)$ becomes

$$
\alpha_{0}(r)=-\dot{C} \sqrt{D(r)} \phi(r) r^{4} \delta(r)^{-3 / 2}+\dot{C} \sqrt{D(r)} \int_{r_{\min }}^{r} \phi(r)\left(r^{4} \delta(r)^{-3 / 2}\right)^{\prime} d r,
$$

where the prime denotes differentiation w.r.t. $r$ in the present context. Note that $\sqrt{D(r)} \phi(r)$ is a regular bounded function so that the first term in (104) is regular and bounded. Using that $d \alpha_{0} / d l=\sqrt{D} d \alpha_{0} / d r$ we obtain by differentiating (104)

$$
\alpha_{0}^{\prime}(L)=\frac{\dot{C}}{2} D^{\prime}\left(r_{\min }\right) \int_{r_{\min }}^{r_{\max }} \phi(r)\left(r^{4} \delta(r)^{-3 / 2}\right)^{\prime} d r=-\frac{\dot{C}}{2} D^{\prime}\left(r_{\min }\right) \int_{r_{\min }}^{r_{\max }} \Phi(r)\left(r^{4} \delta(r)^{-3 / 2}\right)^{\prime \prime} d r
$$


where $\Phi(r)=-4 \sqrt{\left(r-r_{\min }\right)\left(r_{\max }-r\right)}\left(r_{\max }-r_{\min }\right)^{-2}$ is the integral of $\phi(r)$. Therefore, since $\Phi(r)<0$ in $\left(r_{\min }, r_{\max }\right)$, to show that $\alpha_{0}^{\prime}(L) \neq 0$ it is sufficient to show that the function $r^{4} \delta(r)^{-3 / 2}$ is convex.

The special case $C=0$ is easy to treat. In this case, $\delta(r)=(\bar{\Lambda} / 3) r^{3}\left(r-r_{\text {neg }}\right)$, where $r_{\text {neg }}<0$, hence

$$
\left(r^{4} \delta(r)^{-3 / 2}\right)^{\prime \prime} \propto\left(\frac{1}{\sqrt{r\left(r-r_{\mathrm{neg}}\right)^{3}}}\right)^{\prime \prime}=\frac{3\left(r-r_{\mathrm{neg}}\right)^{4}\left(8 r^{2}-4 r_{\mathrm{neg}} r+r_{\mathrm{neg}}^{2}\right)}{4\left[r\left(r-r_{\mathrm{neg}}\right)^{3}\right]^{5 / 2}}>0,
$$

i.e. $r^{4} \delta(r)^{-3 / 2}$ is convex.

In the general case we show that $r^{3} \delta(r)^{-1}$ is convex. Then, $r^{8 / 3} \delta^{-1}=r^{-1 / 3} r^{3} \delta(r)^{-1}$ is convex, since $r^{3} \delta(r)^{-1}$ is decreasing, and consequently $r^{4} \delta(r)^{-3 / 2}=\left(r^{8 / 3} \delta^{-1}\right)^{3 / 2}$ is convex. An in-depth analysis of the properties of the zeros of $\delta(r)$ is essential to establish the claim. Asymptotically, for $C \rightarrow C_{b, t}$, the zeros $r_{\min }$ and $r_{\max }$ of $D(r)$ coincide to form the double zero $r_{-}$, which is know explicitly, see (73). Hence $\delta(r)$ is known explicitly for $C \rightarrow C_{b, t}$,

$$
\begin{aligned}
\delta(r) & =-\frac{r_{0}}{3} \frac{\left(r_{0}-3 M\right)^{2}}{3 r_{0}^{2} V\left(r_{0}\right)} \pi\left(\frac{r}{r_{0}}\right), \\
\text { where } \quad \pi(x) & =1+2 x+3 x^{2}+2 F x^{3}+F x^{4} \quad \text { with } \quad F=1+\frac{3 r_{0}^{2} V\left(r_{0}\right)}{\left(r_{0}-3 M\right)^{2}},
\end{aligned}
$$

and convexity of $r^{3} \delta(r)^{-1}$ can be established; here, $r_{0}$ is such that $\left(K\left(r_{0}\right), C\left(r_{0}\right)\right)$ describes a point on $C_{b, t}$ via (91). Since $\delta(r)$ is a fourth-order polynomial, also its zeros are known explicitly for $C \rightarrow C_{b, t}$. Combining this with an analysis of the variation of the zeros of $\delta$ as $C$ varies, the claim can be established; the details are omitted here.

In order to differentiate (101), i.e. to be able to write down $\alpha_{0}^{\prime}(2 L)$ in terms of quadratures, the integral must be regularized appropriately. The integral representation (101) of $\alpha_{0}$ can be "regularized" in several ways, e.g.

$$
\begin{aligned}
& \alpha_{0}=1-r^{\prime} \int_{0}^{l} \frac{1}{r^{\prime 2}}\left(r_{\min }^{\prime \prime} \frac{r_{\min }^{2}}{r^{2}}-r^{\prime \prime}\right) d l, \\
& \alpha_{0}=\frac{r_{\min }^{2}}{r^{2}}+r_{\min }^{2} r^{\prime} \int_{0}^{l} \frac{2}{r^{3}} d l-r_{\min }^{2} r^{\prime} \int_{0}^{l} \frac{1}{r^{2} r^{\prime 2}}\left(r_{\min }^{\prime \prime}-r^{\prime \prime}\right) d l,
\end{aligned}
$$

where the integrands are now regular at $l=0$. When we differentiate the expression (108b), and manipulate the arising terms so that divergencies cancel, we obtain

$$
\begin{aligned}
\frac{\alpha_{0}^{\prime} r_{\max }^{\prime \prime}}{r_{\min }^{2}}=-\frac{1}{r^{2} r^{\prime}}\left(r_{\max }^{\prime \prime}\right. & \left.-r^{\prime \prime}\right)\left(r_{\min }^{\prime \prime}-r^{\prime \prime}\right)+r^{\prime \prime} \int_{0}^{l} \frac{2}{r^{3}}\left(r_{\min }^{\prime \prime}+r_{\max }^{\prime \prime}-r^{\prime \prime}\right) d l+ \\
& +r^{\prime \prime} \int_{0}^{l} \frac{r^{\prime \prime \prime}}{r^{2} r^{\prime}} d l-r^{\prime \prime} \int_{0}^{l} \frac{1}{r^{2} r^{\prime 2}}\left(r_{\max }^{\prime \prime}-r^{\prime \prime}\right)\left(r_{\min }^{\prime \prime}-r^{\prime \prime}\right) d l,
\end{aligned}
$$


which can be evaluated at $L$ to obtain $\alpha_{0}^{\prime}(L)$ and thus $\alpha_{0}^{\prime}(2 L)$; the equation is mainly useful for numerical purposes.

We consider now the lapse equation

$$
\Delta \alpha+a \alpha=\dot{K}, \quad \text { i.e. } \quad \alpha^{\prime \prime}+\frac{2}{r} r^{\prime} \alpha^{\prime}+a \alpha=\dot{K}=\text { const },
$$

where $a(l)=\Lambda-K^{2} / 3-6 C^{2} / r^{6}(l)$, cf. (38). In first order from the system corresponds to (94) with an additional inhomogeneity $(0, \dot{K})$. Using the principal solution matrix (95) of the homogeneous system, we obtain

$$
\left(\begin{array}{c}
\alpha \\
\alpha^{\prime}
\end{array}\right)=\Pi(l, 0)\left(\begin{array}{c}
\alpha \\
\alpha^{\prime}
\end{array}\right)(0)+\int_{0}^{l} \Pi(l, s)\left(\begin{array}{c}
0 \\
\dot{K}
\end{array}\right) d s
$$

by the method of variation of constants.

Corollary 4.3 states that there exists a unique even periodic solution of (110); we give an alternative argument here. Eq. (111) describes a periodic function if and only if $\left(\alpha, \alpha^{\prime}\right)(2 L)=$ $\left(\alpha, \alpha^{\prime}\right)(0)$, i.e. iff

$$
(\mathrm{id}-\Pi(2 L, 0))\left(\begin{array}{c}
\alpha \\
\alpha^{\prime}
\end{array}\right)(0)=\int_{0}^{2 L} \Pi(2 L, s)\left(\begin{array}{c}
0 \\
\dot{K}
\end{array}\right) d s,
$$

where $\Pi(2 L, 0)$ is given by (97); $\Pi(2 L, s)$ can be computed easily,

$$
\Pi(2 L, s)=\Pi(2 L, 0) \Pi(s, 0)^{-1}=\frac{r(s)^{2}}{r_{\min }^{2}}\left(\begin{array}{cc}
1 & 0 \\
\alpha_{0}^{\prime}(2 L) & 1
\end{array}\right)\left(\begin{array}{cc}
\frac{r^{\prime \prime}(s)}{r_{\min }^{\prime \prime}} & -\frac{r^{\prime}(s)}{r_{\min }^{\prime \prime}} \\
-\alpha_{0}^{\prime}(s) & \alpha_{0}(s)
\end{array}\right) .
$$

Integration of the first component of $\Pi(2 L, s)(0,1)$ yields

$$
\int_{0}^{2 L} \frac{r^{2}}{r_{\min }^{2}}\left(-\frac{r^{\prime}}{r_{\min }^{\prime \prime}}\right) d s=-\left.\frac{1}{r_{\min }^{2} r_{\min }^{\prime \prime}} \frac{r^{3}}{3}\right|_{0} ^{2 L}=0
$$

i.e. the first component of Eq. (112) is satisfied identically. The second condition is a condition for $\alpha(0)$; it is fulfilled iff

$$
\alpha(0)=-\frac{\dot{K}}{\alpha_{0}^{\prime}(2 L)} \frac{1}{r_{\min }^{2}} \int_{0}^{2 L} r(s)^{2} \alpha_{0}(s) d s=: \alpha_{\min } .
$$

Eq. (112) does not impose a condition on $\alpha^{\prime}(0)$; however, when $\alpha$ is required to be even, $\alpha^{\prime}(0)=0$ is necessary. Hence we have reproduced the result that there exists a unique even periodic solution of (110).

The general solution of (110) is the linear combination $\hat{\alpha}=\alpha+k_{0} \alpha_{0}+k_{\xi} \alpha_{\xi}$, where $k_{0}$ and $k_{\xi}$ are constants;

$$
\hat{\alpha}(2 n L+l)=\alpha(l)+k_{0} \alpha_{0}(l)+\left[k_{\xi}+k_{0} n \alpha_{0}^{\prime}(2 L) / r_{\min }^{\prime \prime}\right] \alpha_{\xi}(l) \quad \forall l, \forall n \in \mathbb{Z}
$$


follows from (99). When $k_{0}=0, k_{\xi} \neq 0$, the solution is periodic but not even; when $k_{0} \neq 0$, $k_{\xi}=0$, the solution is even but not periodic.

To express $\alpha_{\min }$, cf. (115), in terms of quadratures we may use the regularization (108b). We obtain

$$
\frac{\alpha_{\min }}{\dot{K}}=-\frac{2}{3 \alpha_{0}^{\prime}(2 L)}\left[L+2 r_{\max }^{3} \int_{0}^{L} \frac{d l}{r^{3}}-\int_{0}^{L} \frac{\left(r_{\max }^{3}-r^{3}\right)\left(r_{\min }^{\prime \prime}-r^{\prime \prime}\right)}{r^{2} r^{\prime 2}} d l\right]-\frac{1}{3} \frac{r_{\min }^{3}-r_{\max }^{3}}{r_{\min }^{\prime \prime}} .
$$

By using (108a) and slightly different conventions we derive

$$
\alpha_{\min }=0 \Leftrightarrow \int_{r_{\min }}^{r_{\max }} \frac{r^{2} d r}{\sqrt{D(r)}}-\frac{1}{6} \int_{r_{\min }}^{r_{\max }} \frac{r_{\min }^{3}-r^{3}}{D^{3 / 2}(r)}\left(D_{\max }^{\prime} \frac{r_{\max }^{2}}{r^{2}}-D^{\prime}(r)\right) d r=0,
$$

where $D^{\prime}(r)=d D(r) / d r$ and $D_{\max }^{\prime}=D^{\prime}\left(r_{\max }\right)$.

We conclude this section by proving the claim made in the proof of Theorem [5.3, i.e. that the system

$$
\Delta \beta+a \beta=\dot{K}, \quad \Delta \beta-3 \frac{r^{\prime}}{r} \beta^{\prime}-\frac{3 b}{r^{3}} \beta=\frac{3 \dot{C}}{r^{3}},
$$

where $a(l)=\Lambda-K^{2} / 3-6 C^{2} / r^{6}(l)$, and $b(l)=M+K C / 3-4 C^{2} / r^{3}(l)$, has a unique even solution $\beta(l)$ (on the domain $\mathbb{R}$ ) for given even functions $\dot{K}$ and $\dot{C}$. It is straightforward to see that the system (118) is equivalent to the equation

$$
r^{\prime} \beta^{\prime}-r^{\prime \prime} \beta=\frac{\dot{K} r}{3}-\frac{\dot{C}}{r^{2}},
$$

which we have encountered already in (52). Since the coefficient $r^{\prime}$ is odd, $r^{\prime \prime}$ even, there exists a unique solution $\beta$ that is even; the general solution $\hat{\beta}$ is a linear combination $\hat{\beta}=\beta+\operatorname{const} r^{\prime}$.

\section{The space $\mathcal{K} \bigodot_{0+}$ and foliations $\mathcal{S}_{\tau}$}

In this section we discuss analytical and numerical results concerning the question of when a compact CMC-slicing $\mathcal{S}_{\tau}$ in the spacetime $\mathrm{KSSdS}[\mathrm{T}]$ is a foliation. These results strengthen the statement of Theorem [5.4.

Let $\alpha(K, C ; l)$ denote the unique even solution of the lapse equation (38) associated with $(K, C) \in \mathcal{K C}_{0}$ and a given constant $\dot{K}>0$, cf. Corollary 4.3. We make the following

Definition. $\mathcal{K}_{0+}$ is defined as the set of all $(K, C) \in \mathcal{K C}_{0}$ such that the associated lapse function $\alpha(K, C ; l)$ is positive.

Proposition C.1. There exists a neighborhood $W$ of the line $C=0$ in $\mathcal{K C}_{0}$ such that

$$
W \subseteq \mathcal{K} \bigodot_{0+}
$$


Proof. The proof is similar to the proof of Theorem [5.4 $\alpha_{\min }(K, C)$ and thus $\alpha(K, C ; l)$ depends continuously on $(K, C) \in \mathcal{K C}_{0}$, when $\dot{K}$ is a given constant (or a continuous function on $\left.\mathcal{K C}_{0}\right)$, see Appendix B. Hence, since $\alpha(K, C ; l)=$ const $>0$ on the line $C=0$, cf. (70), there exists a neighborhood $W$ of $C=0$ which is contained in $\mathcal{K C}_{0+}$.

Proposition C.2. There exists a neighborhood $V\left(C_{b}\right)$ in $\overline{\mathcal{K} \mathcal{C}_{0}}$ of the curve $\left\{\left(K, C_{b}(K)\right) \mid\right.$ $\mid K \in(-\sqrt{3 \Lambda}, \sqrt{3 \Lambda})\}$ and a neighborhood $V\left(C_{t}\right)$ of $\left\{\left(K, C_{t}(K)\right) \mid K \in(-\sqrt{3 \Lambda}, \sqrt{3 \Lambda})\right\}$ such that

$$
V\left(C_{b}\right) \cap \mathcal{K C}_{0+}=\emptyset, \quad V\left(C_{t}\right) \cap \mathcal{K} \mathcal{C}_{0+}=\emptyset .
$$

There exists a neighborhood $U(\sqrt{3 \Lambda})$ of the line $\left\{(\sqrt{3 \Lambda}, C) \mid C \in\left(C_{b}(\sqrt{3 \Lambda}), C_{t}(\sqrt{3 \Lambda})\right)\right\}$ in $\overline{\mathcal{K} \bigodot_{0}}$ and an analogous neighborhood $U(-\sqrt{3 \Lambda})$ of $\left\{(-\sqrt{3 \Lambda}, C) \mid C \in\left(C_{b}(-\sqrt{3 \Lambda}), C_{t}(-\sqrt{3 \Lambda})\right)\right\}$ such that

$$
U(\sqrt{3 \Lambda}) \cap \mathcal{K} \mathcal{C}_{0} \subseteq \mathcal{K C}_{0+}, \quad U(-\sqrt{3 \Lambda}) \cap \mathcal{K} \mathcal{C}_{0} \subseteq \mathcal{K} \mathcal{C}_{0+} .
$$

Proof. In KSSdS[T] consider the foliation of the black hole region by $r=$ const hypersurfaces. Recall that $r=r_{0}=$ const is a CMC-hypersurface with metric $d l^{2}+r_{0}^{2} d \Omega^{2}$ and $K=K_{0}$, $C=C_{0}$ given by (91). The lapse function $\alpha_{r}$ of the $r=$ const foliation at $r=r_{0}$ is given by

$$
\alpha_{r}(l) \equiv \frac{\dot{K}}{a\left(r_{0}\right)}, \quad \text { where } \quad a\left(r_{0}\right)=\Lambda-\frac{K_{0}^{2}}{3}-\frac{6 C_{0}^{2}}{r_{0}^{6}}=\text { const } .
$$

It is a solution of the lapse equation (38), where $r^{\prime}=0$, i.e. $\alpha_{r}$ solves $\alpha_{r}^{\prime \prime}+a\left(r_{0}\right) \alpha_{r}=\dot{K}$. We consider a hypersurface $r=r_{0}$ such that $r_{0}$ lies in the interval given in (92), so that we have $K_{0} \in(-\sqrt{3 \Lambda}, \sqrt{3 \Lambda})$ and (without loss of generality) $C_{0}=C_{t}\left(K_{0}\right)$.

Consider a neighborhood of $\left(K_{0}, C_{0}\right)$ in $\overline{\mathcal{K} \mathcal{C}_{0}}$. Choose a pair $(K, C) \in \mathcal{K}_{0}$ of that neighborhood and consider the associated CMC-hypersurface which is determined by the embedding $t(r)$, see (24). For all small $\epsilon>0$, for all large $E>0$,

$$
\left|t\left(r_{\min }(K, C)+\epsilon\right)\right|=\left|\int_{r_{\min }(K, C)}^{r_{\min }(K, C)+\epsilon} \frac{V^{-1}}{\sqrt{D(K, C ; r)}}\left(\frac{K r}{3}-\frac{C}{r^{2}}\right) d r\right|>E
$$

and $\left|r_{\min }(K, C)-r_{0}\right|<\epsilon$, provided that $(K, C)$ is sufficiently close to $\left(K_{0}, C_{0}\right)$. This follows from (24) using the same techniques as in the proof of Lemma 5.1] and Proposition [5.2. In the subset $t \in[-E, E]$ of the black hole region, the (monotonic) function $r(t)$ thus satisfies $\left|r(t)-r_{0}\right|+|\partial r / \partial t| \leq 3 \epsilon$, if $(K, C)$ is sufficiently close to $\left(K_{0}, C_{0}\right)$, i.e., $r(t)$ is approximately constant for $t \in[-E, E]$.

Let, now, $\mathcal{S}$ be a compact CMC-hypersurface whose values $\left(K_{\mathcal{S}}, C_{\mathcal{S}}\right)$ are sufficiently close to $\left(K_{0}, C_{0}\right)$, i.e. $\left|\left(K_{\mathcal{S}}, C_{\mathcal{S}}\right)-\left(K_{0}, C_{0}\right)\right|<\delta ; \mathcal{S}$ can be approximated by the hypersurface $r=r_{0}$ in a region $t \in[-E, E]$ of the black hole. Consider the compact CMC-slicing $\mathcal{S}_{\tau}$ that contains the CMC-hypersurface $\mathcal{S}$ associated with $\left(K_{\mathcal{S}}, C_{\mathcal{S}}\right)$. Since the oriented direction field on $\mathcal{K C}_{0}$ is tangential to $\partial\left(\mathcal{K C}_{0}\right)$ in the limit $(K, C) \rightarrow\left(K_{0}, C_{0}\right)$, the integral curve $(K, C)(\tau)$ that represents $\mathcal{S}_{\tau}$ can be approximated (in at least the $\mathcal{C}^{1}$-sense) by the curve $\left(K(\tau), C_{t}(K(\tau))\right)$ for all $\tau$ of some (small) $\tau$-interval (independent of $\delta$ ). 
It follows that the above statement carries over to the slicings $\mathcal{S}_{\tau}$ : in some region $t \in[-E, E]$ of the black hole, for all $\tau$ in some small interval, the slicing $\mathcal{S}_{\tau}$ can be approximated by the slicing of $r=$ const hypersurfaces through $r=r_{0}$. We conclude that

$$
\alpha_{\min }(K, C) \rightarrow \alpha_{r}=\frac{\dot{K}}{a\left(r_{0}\right)} \quad \text { for }(K, C) \rightarrow\left(K_{0}, C_{0}\right) .
$$

Standard algebraic manipulations reveal that $a\left(r_{0}\right)<0$ for all $\left(K_{0}, C_{0}\right) \in C_{t}$. Therefore, $\alpha_{\min }(K, C)<0$ for all $(K, C)$ sufficiently close to $C_{t}$, i.e. there exists a neighborhood $V\left(C_{t}\right)$ of the line $\left\{\left(K, C_{t}(K)\right) \mid K \in(-\sqrt{3 \Lambda}, \sqrt{3 \Lambda})\right\}$ in $\overline{\mathcal{K} \bigodot_{0}}$ such that $V\left(C_{t}\right) \cap \mathcal{K} \bigodot_{0+}=\emptyset$. The statement for $C_{b}$ follows via the symmetry property of $\mathcal{K C}_{0}$, hence the first claim is established.

The proof of the second claim of the proposition follows the same principle: we consider a CMC-hypersurface $H_{0}$ in KSSdS that is associated with mean curvature $K_{0}=\sqrt{3 \Lambda}$ and $C_{0} \in\left(C_{b}(\sqrt{3 \Lambda}), C_{t}(\sqrt{3 \Lambda})\right)$ and we exploit the fact that the family of CMC-hypersurfaces $H_{\tau}$ generated by $(\sqrt{3 \Lambda}, C(\tau))$, where $C(\tau)$ is running in $\left(C_{b}(\sqrt{3 \Lambda}), C_{t}(\sqrt{3 \Lambda})\right)$, forms a foliation of (a part of) KSSdS; in particular, $\alpha(r)>0$ for $H_{0}$. The slicing $H_{\tau}$ has been investigated in 6]. However, a proof of the positivity of the associated lapse function is missing. This gap can be closed via considerations similar to those of Appendix B for a detailed discussion see [5].

When $\mathcal{S}$ is a compact CMC-hypersurface whose values $\left(K_{\mathcal{S}}, C_{\mathcal{S}}\right)$ are sufficiently close to $\left(K_{0}=\right.$ $\left.\sqrt{3 \Lambda}, C_{0}\right)$, it can be suitably approximated by the hypersurface $H_{0}$, because $r_{\min }(K, C) \rightarrow$ $r_{\min }\left(\sqrt{3 \Lambda}, C_{0}\right)$ and

$$
t(K, C ; r) \rightarrow t\left(\sqrt{3 \Lambda}, C_{0} ; r\right) \quad \text { when } \quad(K, C) \rightarrow\left(\sqrt{3 \Lambda}, C_{0}\right)
$$

at least in $\mathcal{C}^{1}$, uniformly on every compact $r$-interval. Since the oriented direction field on $\mathcal{K C}_{0}$ is tangential to $\partial\left(\mathcal{K C}_{0}\right)$ in the limit $(K, C) \rightarrow\left(\sqrt{3 \Lambda}, C_{0}\right)$ we infer

$$
\alpha(K, C ; r) \rightarrow \alpha\left(\sqrt{3 \Lambda}, C_{0} ; r\right) \quad \text { for }(K, C) \rightarrow\left(\sqrt{3 \Lambda}, C_{0}\right),
$$

hence, if $(K, C)$ is in a sufficiently small neighborhood of $\left(\sqrt{3 \Lambda}, C_{0}\right)$, then $\alpha(K, C ; r)$ is positive since $\alpha\left(\sqrt{3 \Lambda}, C_{0} ; r\right)$ is positive. Accordingly, there exists a neighborhood $U(\sqrt{3 \Lambda})$ of the line $\left\{(\sqrt{3 \Lambda}, C) \mid C \in\left(C_{b}(\sqrt{3 \Lambda}), C_{t}(\sqrt{3 \Lambda})\right)\right\}$ in $\overline{\mathcal{K}} \bigodot_{0}$ such that $U(\sqrt{3 \Lambda}) \cap \mathcal{K} \bigodot_{0} \subseteq \mathcal{K C}_{0+}$.

Corollary C.3. The compact CMC-slicing $\mathcal{S}_{\tau}, \tau \in\left(\tau_{-}, \tau_{+}\right)$, in the spacetime KSSdS[T], $|T|$ sufficiently large, cannot be a foliation, i.e. $\left(\bar{\tau}_{-}, \bar{\tau}_{+}\right) \neq\left(\tau_{-}, \tau_{+}\right)$in Theorem 5.4.

Proof. The integral curve $(K, C)(\tau)$ associated with the slicing $\mathcal{S}_{\tau}$ is characterized by $\mathcal{T}(\tau) \equiv$ $\mathrm{T}$; when $|\mathrm{T}|$ is sufficiently large, it must pass through a given neighborhood of $C_{b}$ or $C_{t}$ (where $\mathcal{T}= \pm \infty$, cf. Fig. 17) and thus through $V\left(C_{b}\right)$ or $V\left(C_{t}\right)$; in $V\left(C_{b}\right)$ and $V\left(C_{t}\right)$ the lapse function is not positive.

Remark. Let $\mathcal{S}_{\tau}^{\prime}$ be an arbitrary slicing in $\mathrm{KSSdS}[\mathrm{T}]$ that is not reflection symmetric and let $\hat{\alpha}$ denote its lapse function. $\mathcal{S}_{\tau}^{\prime}$ arises from $\mathcal{S}_{\tau}$ by combining the flow of $\mathcal{S}_{\tau}$ with an appropriate admixture of the Killing flow, see Theorem 5.3. therefore $\hat{\alpha}(l)=\alpha(l)+k_{\xi} \alpha_{\xi}(l)=\alpha(l)+k_{\xi} r^{\prime}(l)$ for a constant $k_{\xi}$ (depending on $\tau$ ), see Appendix B Since $r^{\prime}(l)$ is odd, $\hat{\alpha} \ngtr 0$ whenever $\alpha \ngtr 0$, i.e. if $\mathcal{S}_{\tau}$ is not a foliation then there exists no other slicing $\mathcal{S}_{\tau}^{\prime}$ in $\operatorname{KSSdS}[\mathrm{T}]$ that is. 
Numerical investigations suggest that $\mathcal{K C}_{0+}$ is a connected set; the boundary $\partial\left(\mathcal{K C}_{0+}\right)$ consists of two smooth curves: one curve, $P_{t}$, that connects the points $\left(-\sqrt{3 \Lambda}, C_{t}(-\sqrt{3 \Lambda})\right)$ and $\left(\sqrt{3 \Lambda}, C_{t}(\sqrt{3 \Lambda})\right)$, and a second curve, $P_{b}$, that connects the points $\left(-\sqrt{3 \Lambda}, C_{b}(-\sqrt{3 \Lambda})\right)$ and $\left(\sqrt{3 \Lambda}, C_{b}(\sqrt{3 \Lambda})\right)$. The curve $P_{t}$ is given by

$$
P_{t}=\left\{(K, C) \mid\left(\alpha_{\min }(K, C)=0\right) \wedge(C>0)\right\},
$$

cf. (117), $P_{b}$ is the reflected curve, see Figs. 12 and 13. As with $C_{b, t}$ we use the same symbols when we write the curves in parametric form, i.e. we also write $C=P_{b}(K)$ and $C=P_{t}(K)$.

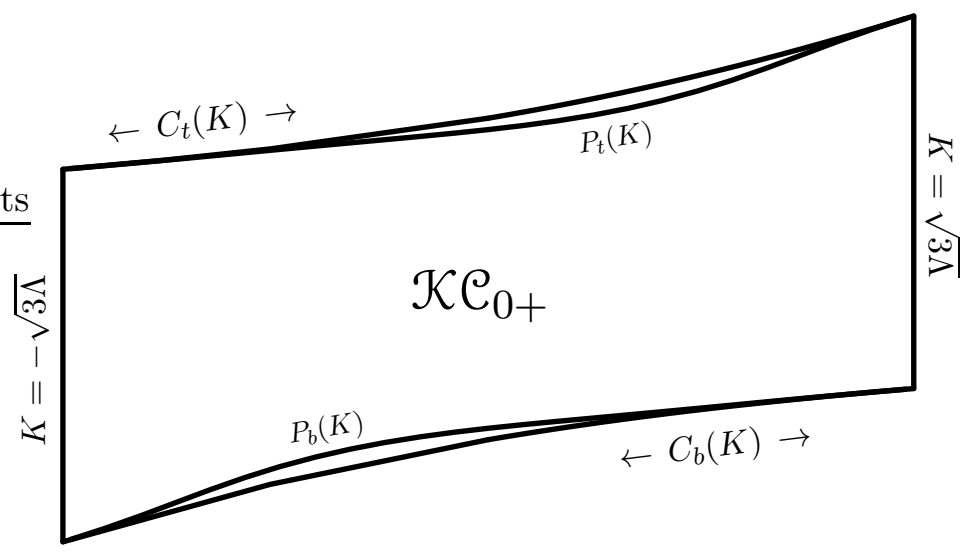

Figure 12: $\mathcal{K C}_{0+}$ and its boundaries $P_{t}, P_{b}$ in the case $\Lambda=1, M=1 / 4$.

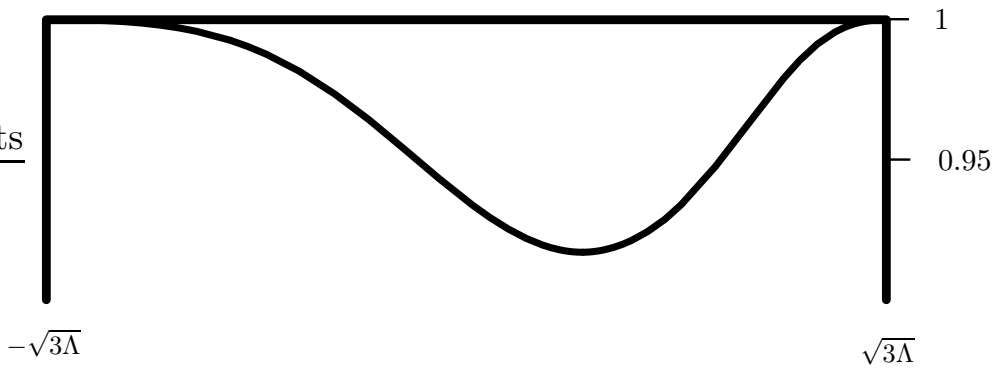

Figure 13: The depicted function represents $P_{t}(K) / C_{t}(K)$.

A slicing $\mathcal{S}_{\tau}, \tau \in\left(\tau_{-}, \tau_{+}\right)$, in $\operatorname{KSSdS}[\mathrm{T}]$ is a foliation as long as the associated integral curve $(K, C)(\tau)$ of the oriented direction field lies in $\mathcal{K C}_{0+}$; if the integral curve intersects $P_{t}$ or $P_{b}, \mathcal{S}_{\tau}$ cannot be a foliation for all $\tau$. Since the integral curve is characterized by $\mathcal{T}(\tau)=\mathcal{T}(K(\tau), C(\tau)) \equiv \mathrm{T}$, at a possible intersection point $\left(K, P_{t}(K)\right), \mathcal{T}\left(K, P_{t}(K)\right)=\mathrm{T}$ must hold, and analogously $\mathcal{T}\left(K, P_{b}(K)\right)=\mathrm{T}$ for $P_{b}$. Therefore, the problem of whether or under what conditions a slicing is a foliation can be investigated by analyzing the function $\mathcal{T}\left(K, P_{t}(K)\right)$ and the equation $\mathcal{T}\left(K, P_{t}(K)\right)=$ T. Let $\mathrm{T}_{\mathrm{f}}:=\inf _{K} \mathcal{T}\left(K, P_{t}(K)\right)$;

i. if $\mathrm{T}_{\mathrm{f}} \ngtr 0$, then, for each $\mathrm{T}$, the slicing $\mathcal{S}_{\tau}$ in $\mathrm{KSSdS}[\mathrm{T}]$ is not a foliation.

ii. If $\mathrm{T}_{\mathrm{f}}>0$, then slicings $\mathcal{S}_{\tau}$ contained in spacetimes $\mathrm{KSSdS}[\mathrm{T}]$ with $|\mathrm{T}|<\mathrm{T}_{\mathrm{f}}$ are foliations; slicings in KSSdS[T] with $|\mathrm{T}| \geq \mathrm{T}_{\mathrm{f}}$ are not. 
To see (i) and (ii), we note that the equation $\mathcal{T}\left(K, P_{t}(K)\right)=\mathrm{T}$ has a solution $K$ iff $\mathrm{T} \geq \mathrm{T}_{\mathrm{f}}$. By virtue of the reflection symmetry in $\mathcal{K C}_{0}, \mathcal{T}\left(K, P_{b}(K)\right)=-\mathcal{T}\left(-K, P_{t}(-K)\right)$, thus the equation $\mathcal{T}\left(K, P_{b}(K)\right)=\mathrm{T}$ is equivalent to the equation $\mathcal{T}\left(-K, P_{t}(-K)\right)=-\mathrm{T}$; it has a solution iff $\mathrm{T} \leq-\mathrm{T}_{\mathrm{f}}$. If $\mathrm{T}_{\mathrm{f}} \leq 0$, then, for all $\mathrm{T}$, at least one of the equations has a solution. If $\mathrm{T}_{\mathrm{f}}>0$, then, for all $|\mathrm{T}| \geq \mathrm{T}_{\mathrm{f}}$, at least one of the equations has a solution; however, for all $|\mathrm{T}|<\mathrm{T}_{\mathrm{f}}$, neither of the equations has a solution. This implies that the integral curve $\mathcal{T}(\tau)=\mathrm{T}$ (with $|\mathrm{T}|<\mathrm{T}_{\mathrm{f}}$ ) in $\mathcal{K C}_{0}$ does neither intersect $P_{t}$ nor $P_{b}$, but is entirely contained in $\mathcal{K C}_{0+}$; thus the associated slicing $\mathcal{S}_{\tau}, \tau \in\left(\tau_{-}, \tau_{+}\right)$, is a foliation.

Lemma 5.1 entails that $\mathcal{T}\left(K, P_{t}(K)\right) \rightarrow \infty$ for $K \rightarrow-\sqrt{3 \Lambda}$; moreover, the numerical results suggest that the function $\mathcal{T}\left(K, P_{t}(K)\right)$ is always strictly monotonically decreasing for $K<0$. However, for $K>0$, the properties of $\mathcal{T}\left(K, P_{t}(K)\right)$ depend on the chosen family of KSSdS[T]spacetimes, i.e. on $(\Lambda, M)$ ! In Fig. 14] we show the function $\mathcal{T}\left(K, P_{t}(K)\right)$ for several cases of $M$, where $\Lambda=1$.

Numerical results suggest that the asymptotic behavior of $\mathcal{T}\left(K, P_{t}(K)\right)$ as $K \rightarrow \sqrt{3 \Lambda}$ can be approximated by

$$
\mathcal{T}\left(K, P_{t}(K)\right)=c_{1}+c_{2} \log (\sqrt{3 \Lambda}-K),
$$

where the constants $c_{1}, c_{2}$ depend on $(\Lambda, M)$. This is consistent with the asymptotics of $\mathcal{T}$ obtained in the proof of Lemma 5.1. The constant $c_{2}$ is positive (so that $\mathcal{T}\left(K, P_{t}(K)\right) \rightarrow-\infty$ as $K \rightarrow \sqrt{3 \Lambda}$ ) for all $M$ satisfying $M<M_{\mathrm{c}} ; c_{2}=0$ for $M=M_{\mathrm{c}}$, and $c_{2}<0$ in the case $M_{\mathrm{c}}<M<(3 \sqrt{\Lambda})^{-1}$, cf. Fig. 14]

In the case $M \geq M_{\mathrm{c}}$, positivity of $\mathcal{T}\left(K, P_{t}(K)\right)$ is possible; indeed, there exists $M_{0}$ such that $\mathrm{T}_{\mathrm{f}}>0$ for all $M_{\mathrm{c}} \leq M<M_{0}$, see Figs. 14(d) and 14(e). Hence, for $M_{\mathrm{c}} \leq M<M_{0}$, the slicings $\mathcal{S}_{\tau}$ in the spacetimes $\mathrm{KSSdS}[\mathrm{T}]$ whose $|\mathrm{T}|$ is small enough, are foliations, cf. (ii). Thus there is strong numerical evidence that Conjecture [5.5] is true.

From Fig. 14(c) we see that the equation $\mathcal{T}\left(K, P_{t}(K)\right)=\mathrm{T}$ can have up to three solutions. Since in that case $\mathcal{T}\left(K, P_{b}(K)\right)=\mathrm{T}$ has also one solution, an integral curve of the oriented direction field can switch between $\mathcal{K C}_{0+}$ and $\mathcal{K C}_{0} \backslash \mathcal{K} \mathcal{C}_{0+}$ up to four times. The different scenarios can be read off from Fig. 14.

We conclude this section by noting that the asymptotic behavior of $P_{t}(K)$ is given by

$$
P_{t}(K)=C_{t}(K)\left(1-\left[d_{1}+d_{2}(\sqrt{3 \Lambda}-K)\right](\sqrt{3 \Lambda}-K)^{2}\right)
$$

where $d_{1}$ and $d_{2}$ are constants, $d_{1}>0$, that depend on $(M, \Lambda)$.

\section{References}

[1] L. Andersson. The Global Existence Problem in General Relativity. In P.T. Chruściel and H. Friedrich, editors, The Einstein Equations and the Large Scale Behavior of Gravitational Fields, pages 71-120. Birkhäuser, 2004.

[2] R. Beig and P.T. Chruściel. Killing initial data. Class. Quantum Grav., 14 : A83-A92, 1997. 


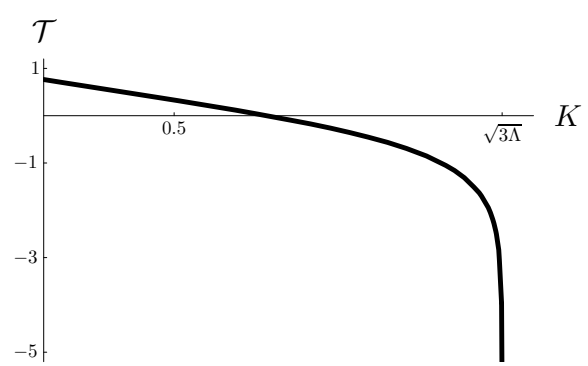

(a) $M=0.100$

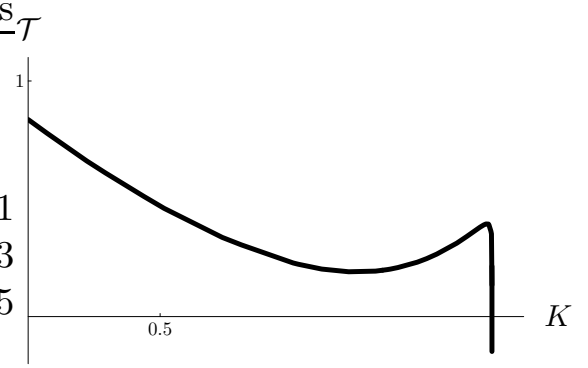

(c) $M=0.215$

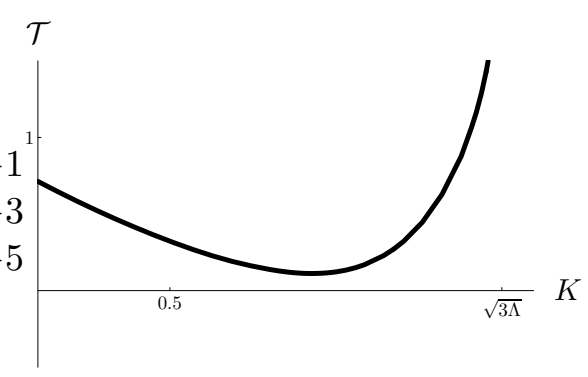

(e) $M=0.250$

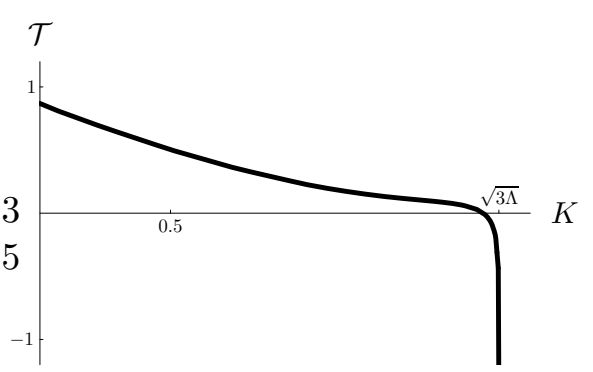

(b) $M=0.200$

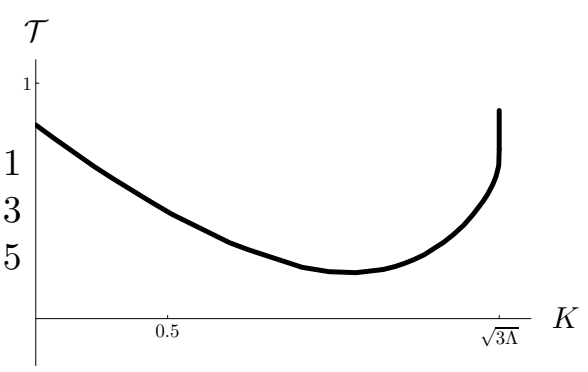

(d) $M=0.220$

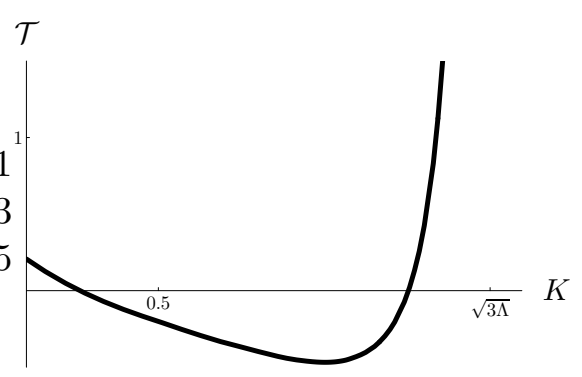

(f) $M=0.330$

Figure 14: The figures show the function $\mathcal{T}\left(K, P_{t}(K)\right)$ for $K>0$ for several values of $M ; \Lambda=1$ in all cases. $\mathcal{T}\left(K, P_{t}(K)\right) \rightarrow-\infty(K \rightarrow \sqrt{3 \Lambda})$ for all $M<M_{\mathrm{c}}$, i.e. for (a) $-(\mathrm{c}) ; \mathcal{T}\left(K, P_{t}(K)\right) \rightarrow+\infty$ $(K \rightarrow \sqrt{3 \Lambda})$ for all $M>M_{\mathrm{c}}$, i.e. for (d)-(f); in the case $M=M_{\mathrm{c}}$ the function converges to a constant; $M_{\mathrm{c}} \approx 0.218945$. $\mathcal{T}\left(K, P_{t}(K)\right) \geq$ const $>0$ for all $K$ when $M \in\left[M_{\mathrm{c}}, M_{0}\right)$, i.e. for (d) and (e); $M_{0} \approx 0.268516$. 
[3] R. Beig and N. Ó Murchadha. Late time behavior of the maximal slicing of the Schwarzschild black hole. Phys. Rev. D, 57 (8): 4728-4737, 1998.

[4] P.T. Chruściel and E. Delay. On mapping properties of the general relativistic constraints operator in weighted function spaces, with applications. Mem. Soc. Math. France, 94 :1103, 2003.

[5] J.M. Heinzle. CMC-Slicings of Kottler-Schwarzschild-de Sitter Cosmologies II. In preparation.

[6] K.-i. Nakao, K.-i. Maeda, T. Nakamura, and K.-i. Oohara. Constant-mean-curvature slicing of the Schwarzschild-de Sitter space-time. Phys. Rev. D, 44 : 1326-1329, 1991.

[7] F. Kottler. Über die physikalischen Grundlagen der Einsteinschen Gravitationstheorie. Ann. Phys. (Germany), 56 : 401-462, 1018.

[8] M. Renardy and R.C. Rogers. An Introduction to Partial Differential Equations. Springer, 2004.

[9] A.D. Rendall. Constant mean curvature foliations in cosmological spacetimes. Helv. Phys. Acta, 69 : 490-500, 1996.

[10] C. Stanciulescu. Spherically Symmetric Solutions of the Vacuum Einstein Field Equations with Positive Cosmological Constant. Master's thesis, University of Vienna, 1998.

[11] T. Valent. Boundary Value Problems of Finite Elasticity. Springer, New York, 1987. 